\title{
The LHCf detector at the CERN Large Hadron Collider
}

The LHCf Collaboration

ABSTRACT: LHCf is an experiment dedicated to the measurement of neutral particles emitted in the very forward region of LHC collisions. The physics goal is to provide data for calibrating the hadron interaction models that are used in the study of Extremely High-Energy Cosmic-Rays. This is possible since the laboratory equivalent collision energy of LHC is $10^{17} \mathrm{eV}$. Two LHCf detectors, consisting of imaging calorimeters made of tungsten plates, plastic scintillator and position sensitive sensors, are installed at zero degree collision angle $\pm 140 \mathrm{~m}$ from an interaction point (IP). Although the lateral dimensions of these calorimeters are very compact, ranging from $20 \mathrm{~mm} \times 20 \mathrm{~mm}$ to $40 \mathrm{~mm} \times 40 \mathrm{~mm}$, the energy resolution is expected to be better than $6 \%$ and the position resolution better than $0.2 \mathrm{~mm}$ for $\gamma$-rays with energy from $100 \mathrm{GeV}$ to $7 \mathrm{TeV}$. This has been confirmed by test beam results at the CERN SPS. These calorimeters can measure particles emitted in the pseudo rapidity range $\eta>8.4$. Detectors, data acquisition and electronics are optimized to operate during the early phase of the LHC commissioning with luminosity below $10^{30} \mathrm{~cm}^{-2} \mathrm{~s}^{-1}$. LHCf is expected to obtain data to compare with the major hadron interaction models within a week or so of operation at luminosity $\sim 10^{29} \mathrm{~cm}^{-2} \mathrm{~s}^{-1}$. After $\sim 10$ days of operation at luminosity $\sim 10^{29} \mathrm{~cm}^{-2} \mathrm{~s}^{-1}$, the light output of the plastic scintillators is expected to degrade by $\sim 10 \%$ due to radiation damage. This degradation will be monitored and corrected for using calibration pulses from a laser.

KEYWORDS: Photon detectors for UV, visible and IR photons; Scintillators, scintillation and light emission processes; Solid state detectors; Calorimeters; Gamma detectors; Particle identification methods; Particle tracking detectors; Photon detectors for UV, visible and IR photons; Gamma detectors; Particle detectors; Radiation damage to detector materials; Data acquisition concepts; Detector control systems; Front-end electronics for detector readout; Trigger concepts and systems; Analysis and statistical methods; Pattern recognition, cluster finding, calibration and fitting methods; Simulation methods and programs; Scintillators and scintillating fibers and light guides; Detector alignment and calibration methods; Overall mechanics design. 


\section{The LHCf Collaboration}

O. Adriani, ${ }^{a}$ L. Bonechi, ${ }^{a}$ M. Bongi, ${ }^{b}$ G. Castellini,${ }^{c}$ R. D'Alessandro, ${ }^{a}$ D.A. Faus, ${ }^{d}$ K. Fukui, ${ }^{e}$ M. Grandi, ${ }^{b}$ M. Haguenauer, ${ }^{f}$ Y. Itow, ${ }^{e}$ K. Kasahara, ${ }^{g}$ D. Macina, ${ }^{h}$ T. Mase,${ }^{e}$ K. Masuda ${ }^{e}$ Y. Matsubara, ${ }^{e}$ H. Menjo, ${ }^{e}$ M. Mizuishi, ${ }^{g}$ Y. Muraki, ${ }^{i}$ P. Papini, ${ }^{b}$ A.L. Perrot, ${ }^{h}$ S. Ricciarini, ${ }^{b}$ T. Sako, ${ }^{e}$ Y. Shimizu, ${ }^{g}$ K. Taki, ${ }^{e}$ T. Tamura, ${ }^{j}$ S. Torii, ${ }^{g}$ A. Tricomi, ${ }^{k}$ W.C. Turner, ${ }^{l}$ J. Velasco, ${ }^{d}$ A. Viciani, ${ }^{b}$ H. Watanabe ${ }^{e}$ and K. Yoshida ${ }^{m}$

${ }^{a}$ Università degli Studi di Firenze and INFN Sezione di Firenze, Firenze, Italy ${ }^{b}$ INFN Sezione di Firenze, Firenze, Italy ${ }^{c}$ IFAC CNR and INFN Sezione di Firenze, Firenze, Italy

${ }^{d}$ IFIC, Centro Mixto CSIC-UVEG, Valencia, Spain

${ }^{e}$ Solar-Terrestrial Environment laboratory, Nagoya University, Nagoya, Japan

${ }^{f}$ Ecole-Polytechnique, Paris, France

${ }^{g}$ Research Institute for Science and Engineering, Waseda University, Tokyo, Japan

${ }^{h}$ CERN, Geneva, Switzerland

${ }^{i}$ Konan University, Kobe, Japan

${ }^{j}$ Kanagawa University, Yokohama, Japan

${ }^{k}$ Università degli Studi di Catania and INFN Sezione di Catania, Catania, Italy

${ }^{l}$ LBNL, Berkeley, California, USA

${ }^{m}$ Shibaura Institute of Technology, Saitama, Japan

Corresponding authors:

Daniela Macina (Daniela.Macina@cern.ch) and Yoshitaka Itow (itow@stelab.nagoya-u.ac.jp) 


\section{Contents}

The LHCf Collaboration ii

1 Introduction 1

1.1 Physics of the LHCf experiment 1

1.2 Experimental overview 3

2 The detectors 6

2.1 Detector overview 6

2.2 Calorimeter 6

$\begin{array}{lll}2.3 & \text { SciFi and MAPMT } & 10\end{array}$

2.4 Silicon strip detector $\quad 11$

$\begin{array}{lll}2.5 & \text { Manipulator } & 14\end{array}$

$\begin{array}{lll}2.6 & \text { Front counters } & 14\end{array}$

3 Trigger and DAQ electronics 16

$\begin{array}{ll}3.1 \text { Global flow of trigger and DAQ } & 16\end{array}$

$\begin{array}{ll}\text { 3.2 Electronics and DAQ for the SciFi detector } & 17\end{array}$

$\begin{array}{ll}3.3 & \text { Electronics and DAQ for the silicon tracking detector . }\end{array}$

4 Performance of the LHCf detectors 19

4.1 Expected performance of the LHCf detectors 19

4.2 Results of SPS beam tests 24

4.2.1 Results for the calorimeters 24

$\begin{array}{ll}\text { 4.2.2 Position resolution of the SciFi } & 27\end{array}$

4.2.3 Position resolution of the silicon tracker 28

4.2.4 Demonstration of $\pi^{0}$ mass reconstruction capability 29

4.3 Results of radiation damage tests 31

5 Summary 33

$\begin{array}{ll}\text { Bibliography } & 35\end{array}$ 


\section{Chapter 1}

\section{Introduction}

\subsection{Physics of the LHCf experiment}

Research on the highest energy cosmic-rays (energy above $10^{19} \mathrm{eV}$ ) has great scientific interest since their origin, propagation and interactions are unknown and may yield information about new physics. To give an idea of the type of information available we briefly summarize the situations with the spectrum and composition of the highest energy cosmic rays and then describe how the LHCf experiment can make a contribution to this field.

Ten years have passed since the air shower experiment AGASA reported that the cosmic ray spectrum at the highest energy may extend beyond $10^{20} \mathrm{eV}$ and the GZK cut-off might not exist [1]. If this were true, the origin of the highest energy cosmic-rays would need an exotic explanation like the decay of cosmic strings or $\mathrm{Z}_{0}$ bursts [2] and so on. However another result obtained by the HiRes experiment was consistent with the existence of the GZK cut-off [3]. The AGASA experiment used a large array of surface detectors whereas the HiRes experiment employed a novel calorimetric technique utilizing observation of atmospheric fluorescence. Recently new results have been published by the Auger collaboration at the international cosmic-ray conference ICRC07 [4]. Auger employs a combination of surface detectors and atmospheric fluorescence telescopes and the new results support the existence of the GZK cut-off. The results of these three experiments are shown in figure 1.1. If the energy scale of the AGASA results were reduced by $45 \%$ then their results would be in agreement with the others.

Measurement of the chemical composition of the highest energy cosmic rays can also give important information about where they are produced. Several astronomical candidates for the production of the highest energy cosmic rays have been discussed in the literature and the maximum energy obtained depends on the composition to be accelerated. The highest energy cosmic-rays can travel through nearby inter-galactic space almost without deviation by magnetic fields. Auger has reported that the arrival direction of the highest energy events is correlated with the distribution of active galactic nuclei (AGN) [5]. This naturally suggests that the primaries are most likely protons. However composition measurements reported by Auger indicate that the primary composition is a mixture of protons and heavy nuclei $(\mathrm{Fe})$ up to the highest energy where they so far have sufficient statistics for composition analysis $-4 \times 10^{19} \mathrm{eV}[6]$. 


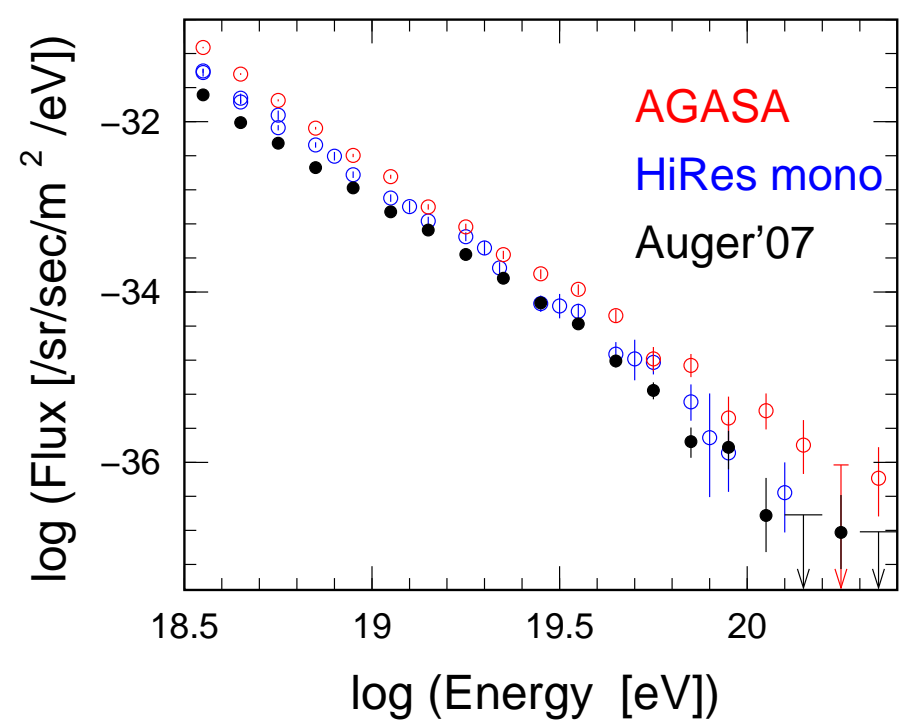

Figure 1.1: Energy spectra of primary cosmic-rays obtained by three large experiments. The red, blue and black points represent the result of AGASA, HiRes and Auger, respectively.

If it turns out that the highest energy primaries have significant Fe composition then the "GZK-cut off"-like feature could perhaps be interpreted as photo-dissociation by cosmic microwave background $(\mathrm{CMB})$ photons. Further investigations are needed for a consistent understanding of all measurements.

It is well known that understanding the results of cosmic ray experiments depends strongly on the use of Monte Carlo codes. However the hadronic interaction models used in these codes have not been verified experimentally near the GZK energy region $10^{20} \mathrm{eV}$ which is 6 orders of magnitude higher than the laboratory equivalent energy of the $\mathrm{S} p \bar{p} \mathrm{~S}$ or Tevatron. The perturbative QCD models used for simulation of air shower experiments are essentially phenomenological models calibrated with experimental data where they are available. So far only data from the UA7 experiment taken at the $\mathrm{S} p \bar{p} \mathrm{~S}$ have been available for calibration of the forward neutral pion production spectrum at $10^{14} \mathrm{eV}$ [9]. The $14 \mathrm{TeV}$ center of momentum energy of the Large Hadron Collider (LHC) will push the laboratory equivalent collision energy up to $10^{17} \mathrm{eV}$.

There are two key quantities at the primary interaction vertices which determine the development of air showers; the total inelastic cross section and the particle production energy spectra at very forward angles. The former will be measured at LHC with roman pot detectors such as employed by the TOTEM [7] or ATLAS collaborations [8]. Therefore a new measurement on forward production spectra in LHC is strongly desired to calibrate the models at $10^{17} \mathrm{eV}$. LHCf is an experiment to perform a measurement of the very forward production cross sections and energy spectra of neutral pions and neutrons. Measurement will be done in a short period during the early phase of the LHC commissioning before the luminosity reaches $10^{30} \mathrm{~cm}^{-2} \mathrm{~s}^{-1}$. In this paper, an overview of the LHCf instrumentation is given. Previous study of the prototype detectors is found elsewhere [10]. After providing an outline of the LHCf experiment in section 1.2, the details of the detectors and the data acquisition system will be described in section 2 and section 3, respectively. In section 4, simulation studies of the expected performance during the LHC operation and the first results from beam tests performed in 2007 at the CERN SPS are reported. 


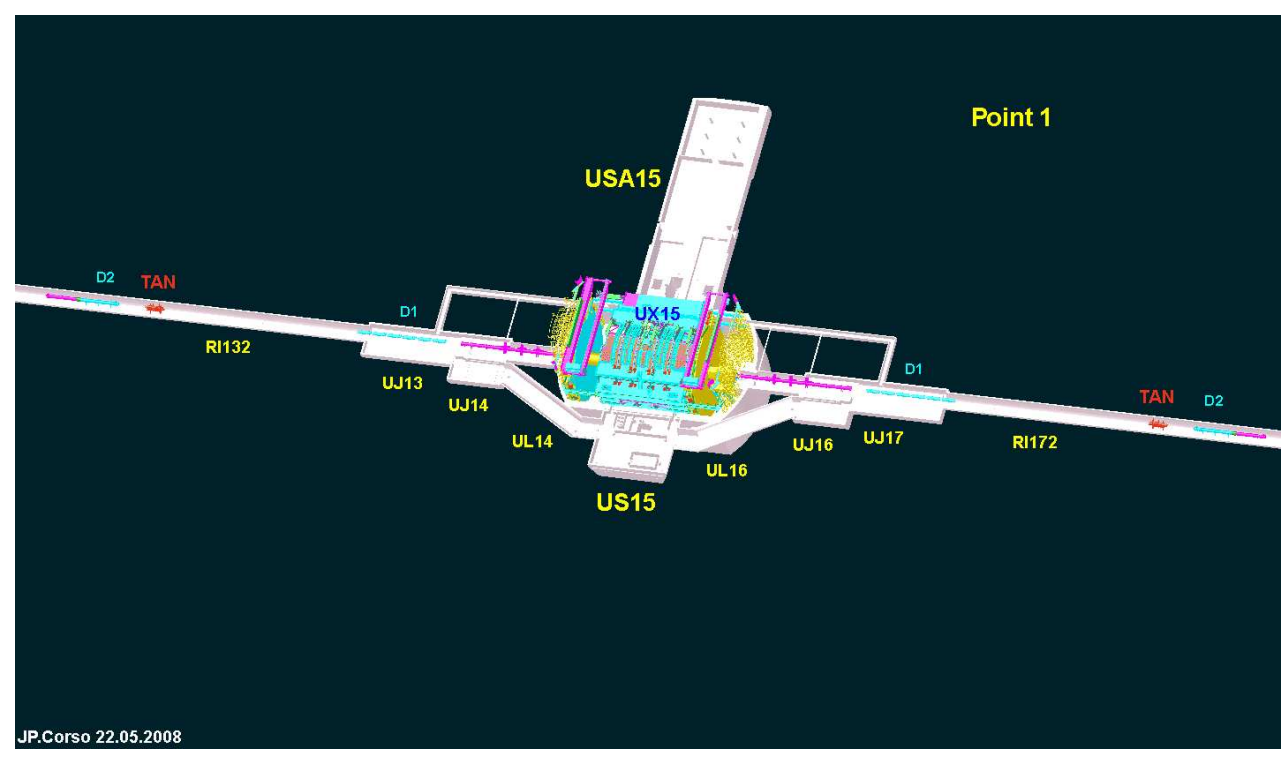

Figure 1.2: Geometry of the IP1 area of LHC. The structure seen in the center represents the ATLAS detector surrounding the interaction point. The straight line from top-left to bottom-right indicates the long straight section of the LHC tunnel and the LHCf detectors are installed at the places marked 'TAN' at both sides of IP1.

\subsection{Experimental overview}

The LHC has massive zero degree neutral absorbers (Target Neutral Absorber; TAN) located $\pm 140 \mathrm{~m}$ from interaction points (IP) 1 and 5 in order to protect the outer superconducting beam separation dipoles (D2) from neutral particle debris from the IP (figure 1.2, figure 1.3). Charged particles from the IP are swept aside by the inner beam separation dipole D1 before reaching the TAN. Inside TAN the beam vacuum chamber makes a Y shaped transition from a single common beam tube facing the IP to two separate beam tubes joining to the arcs of LHC. The Y-chamber has been carefully machined to have a uniform one radiation length projected thickness over a $100 \mathrm{~mm} \times 100 \mathrm{~mm}$ square centered on the zero degree crossing angle beamline. In the crotch of this "Y-chamber", just behind the $100 \mathrm{~mm} \times 100 \mathrm{~mm}$ square there is an instrumentation slot of $96 \mathrm{~mm}^{w} \times$ $607 \mathrm{~mm}^{h} \times 1000 \mathrm{~mm}^{l}$ extending from $67 \mathrm{~mm}$ below the beam height to the top of the TAN. The aperture for the LHCf measurements is limited by the width of the slot and by the vertical aperture of the beam pipe in the D1 dipole projected to the TAN. The cross sections of the D1 beam pipe projected to the detector plane and of the instrumentation slot of the TAN are drawn in figure 1.4. This unique location covers the pseudo-rapidity range from 8.4 to infinity.

The LHCf detectors are two independent shower calorimeters inserted in the TAN instrumentation slots on both sides of IP1. Each occupies a $300 \mathrm{~mm}$ length in the most upstream position of the instrumentation slots followed by BRAN luminosity monitors $100 \mathrm{~mm}$ in length [12] and finally the ATLAS ZDCs [11]. Both the LHCf detectors consist of a pair of small sampling and imaging calorimeters made of plastic scintillators interleaved with tungsten converters. Position sensitive layers are inserted in order to provide incident shower positions. The two detectors are similar, but use different techniques and geometry for the purposes of redundancy and consistency checks of 

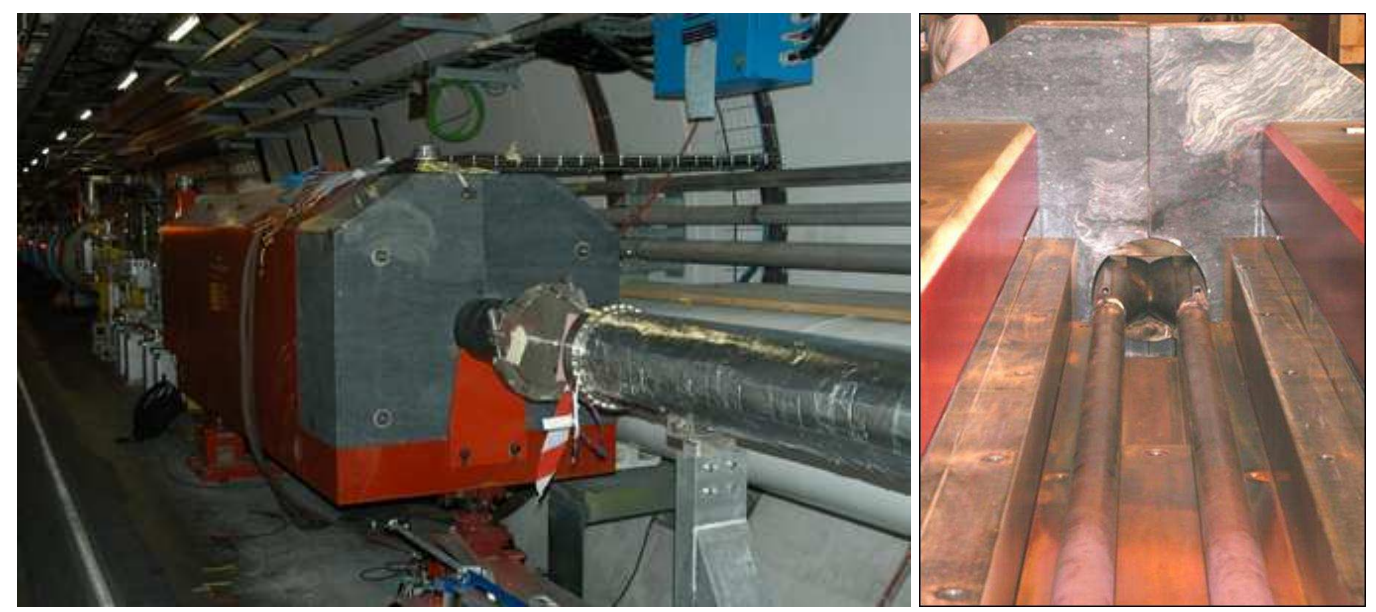

Figure 1.3: Photo of the TAN absorber located $140 \mathrm{~m}$ from the IP. Left: TAN fully assembled in the LHC tunnel seen from the IP side. Right: TAN during assembly at CERN seen from the top facing the IP. The $96 \mathrm{~mm}$ gap between the two beam pipes allows space for installation of detectors.

the measurements. In addition, coincidence between these detectors may be useful for rejection of background due to beam-gas interactions and for application to diffractive physics. The calorimeters are designed to have energy and position resolutions better than $5 \%$ and $0.2 \mathrm{~mm}$, respectively. With such properties, the experiment will be able to discriminate between the major interaction models used in cosmic-ray studies, or to construct new models. In this paper, the detector installed in LSS1L (Arm 1; IP8 side) is referred as detector 1 and the detector installed in LSS1R (Arm 2; IP2 side) is referred as detector 2. Standing inside the LHC ring and looking at IP1, detector 1 is on the left and detector 2 is on the right.

Both detectors are supported by manipulators mounted to the top surface of the TAN in order to have the capability of remotely moving the detectors vertically by a $120 \mathrm{~mm}$ stroke. Figure 1.4 shows the geometrical configuration of each detector viewed from IP1. In default setting, the center of the smaller calorimeters is placed on the horizontal midplane. Using the manipulators to move the detectors vertically from their default positions increases the range of $\mathrm{P}_{T}$ that can be measured. The $\mathrm{P}_{T}$ range would be further increased by operation with a non-zero beam crossing angle of $140 \mu \mathrm{rad}$, because the center of neutral particle flux moves downward. The geometry of detector 2 is designed to maximize the $\mathrm{P}_{T}$ coverage without scanning and/or employing a finite crossing angle. In front of each detector, a Front Counter (FC) made of plastic scintillators is inserted. They provide useful trigger information by covering a larger aperture than the calorimeters.

With these detectors we will be able to identify $\gamma$-rays, measure the $\gamma$-ray energy spectrum $(>100 \mathrm{GeV})$ with a few per cent energy resolution, measure the $\gamma$-ray incident position and reconstruct the $\gamma$-ray pair invariant mass distribution that shows a clear peak at the neutral pion mass. Hadron showers of high energy neutrons at zero degrees can be also measured, however, with decreased energy resolution of about $30 \%$.

LHCf is not designed to be a radiation hard detector and so will be removed when the LHC luminosity exceeds $10^{30} \mathrm{~cm}^{-2} \mathrm{~s}^{-1}$. Owing to the limitations imposed by radiation and the configuration of the DAQ, data taking of LHCf is planed for the 43 bunch operation and $10^{10}$ protons per 


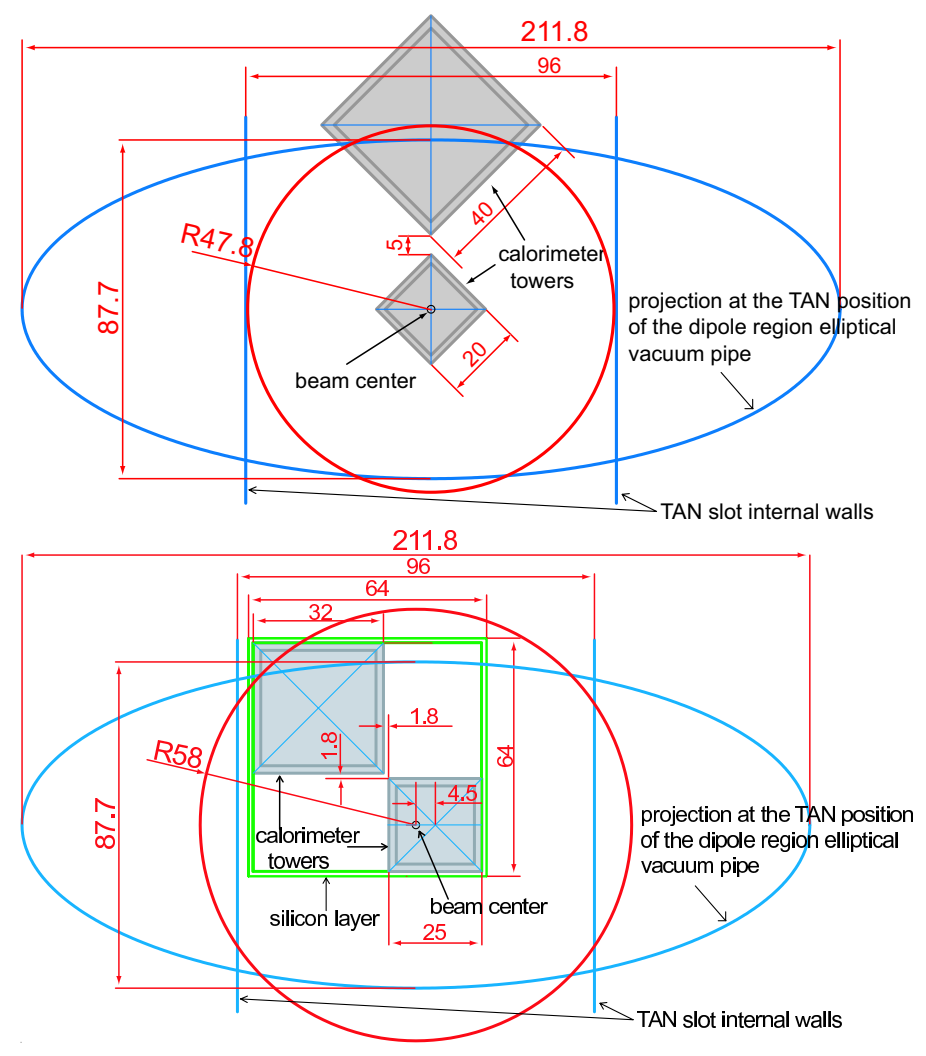

Figure 1.4: Cross sections of detector 1 (top) and detector 2 (bottom). Grey squares indicate the calorimeters in the detectors while a green square shows the coverage of the silicon strip sensor. Vertical and elliptical blue lines indicate the physical aperture limited by the walls of the TAN and the beam pipe, respectively.

bunch which are foreseen at the very beginning of LHC commissioning. At this low intensity and luminosity $\left(10^{29} \mathrm{~cm}^{-2} \mathrm{~s}^{-1}\right)$ a few minutes of data taking can provide enough statistics to discriminate between hadron interaction models. After a week or so of operation it is anticipated that the LHCf detector will be removed from TAN during a brief machine stop. In the absence of LHCf three $\mathrm{Cu}$ bars (each $94 \mathrm{~mm}^{w} \times 605 \mathrm{~mm}^{h} \times 99 \mathrm{~mm}^{l}$ ) will occupy the region in front of the BRAN. The purpose of the $\mathrm{Cu}$ bars is to generate showers for detection by the BRAN as well as to provide shielding for the downstream D2 magnets. The LHCf calorimeters and the Cu bars have nearly the same length in nuclear interaction lengths so the BRAN signals will be very similar for operation with LHCf and with the $\mathrm{Cu}$ bars. 


\section{Chapter 2}

\section{The detectors}

\subsection{Detector overview}

Both LHCf detectors employ two imaging shower calorimeters. While the structure of the calorimeters is similar (except for their sizes and orientation), the position sensitive sensors are quite different. Detector 1 uses scintillating fibers (SciFi) and Multi-anode PMTs (MAPMTs), while detector 2 uses silicon strip sensors. Four X-Y pairs of the position sensors are installed at $6,10,30$ and $42 \mathrm{X}_{0}$ for detector 1 and $6,12,30$ and $42 \mathrm{X}_{0}$ for detector 2 . The first two pairs are optimized to detect the shower maximum of gamma-ray induced showers and the other two are for hadronic showers developed deep in the calorimeters. These position sensitive sensors are necessary not only to obtain the transverse momentum of the incident primary but also to correct for the effect of leakage from the edges of the calorimeters. Because the fraction of shower leakage with respect to the total energy is only a function of the position and independent of the energy, we can correct for this effect by measuring the position of the shower. Details of the shower leakage correction are found elsewhere [10].

Calorimeter components (scintillator, tungsten and PMT), position sensors and their frontend electronics are packed in a $92 \mathrm{~mm}^{w} \times 620 \mathrm{~mm}^{h} \times 280 \mathrm{~mm}^{l}$ aluminum box for each detector as shown in figure 2.1, 2.2, 2.3. The size is designed to fit the narrow instrumentation slot in the TAN. One wall of the box for detector 2 is made of copper to better dissipate the heat generated by the front-end circuitry for the silicon strips. The detectors in their boxes are attached to and supported by the manipulators. In front of each detector (facing the IP), a counter composed of thin plastic scintillators called the Front Counter (FC) is installed.

\subsection{Calorimeter}

The longitudinal (along the beam direction) structure of the calorimeter is shown in figure 2.4. Each calorimeter consists of 16 layers of tungsten plates interleaved with $3 \mathrm{~mm}$ thick plastic scintillators (Eljen Technology EJ-260) for measuring the deposited energy. The thickness of the tungsten plates is $7 \mathrm{~mm}$ for the first 11 layers and $14 \mathrm{~mm}$ for the rest. Including the position sensors the total length is $220 \mathrm{~mm}$. In units of radiation and hadron interaction lengths the total length of a calorimeter is $44 \mathrm{X}_{0}$ and $1.7 \lambda$, respectively. Most of the tungsten layers are attached to holders made of G10 

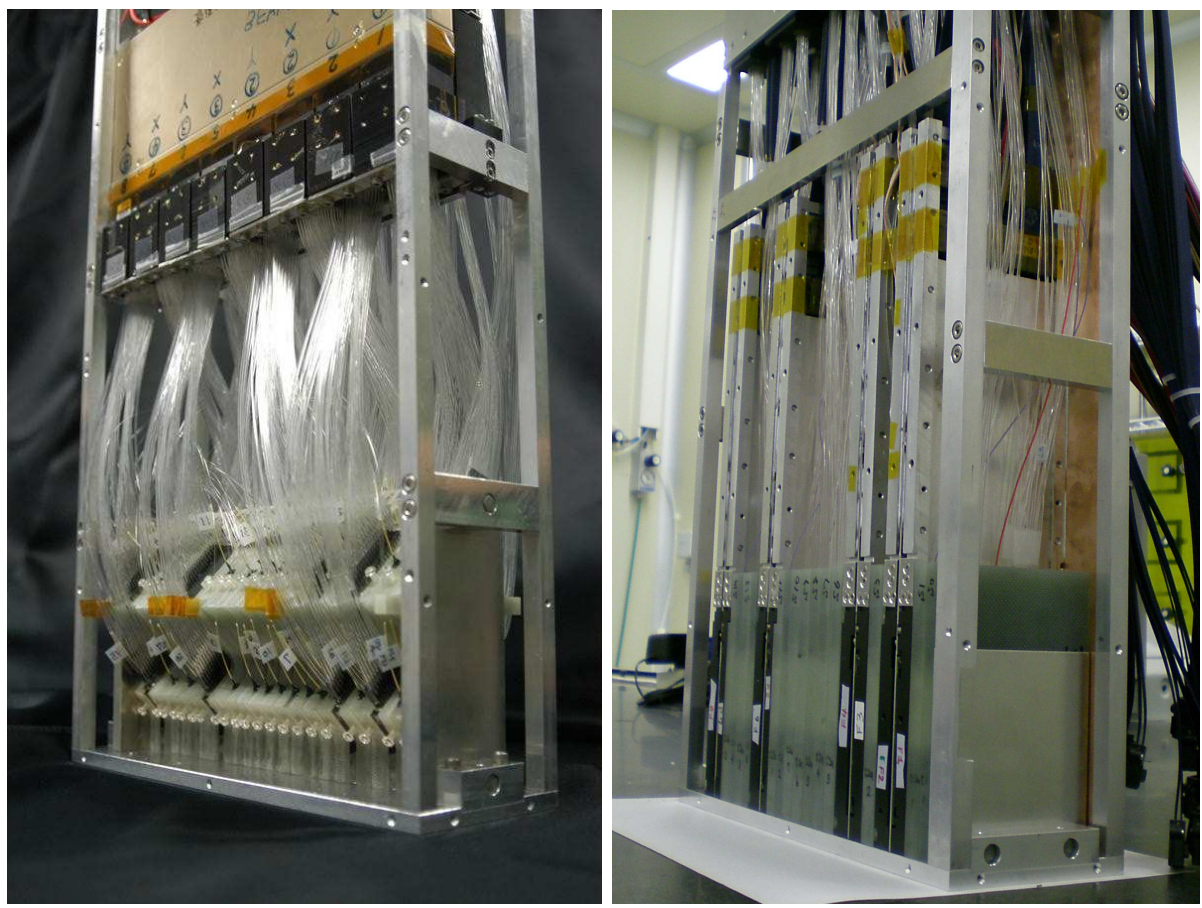

Figure 2.1: Photo of detector 1 (left) and detector 2 (right).

while the holders near the silicon layers are made of Delrin. All the layers are stacked together and pinned in position with two G10 rods. The transverse sizes of the calorimeters are $20 \mathrm{~mm} \times 20 \mathrm{~mm}$ and $40 \mathrm{~mm} \times 40 \mathrm{~mm}$ for detector 1 and $25 \mathrm{~mm} \times 25 \mathrm{~mm}$ and $32 \mathrm{~mm} \times 32 \mathrm{~mm}$ for detector 2 . The cross sections of two detectors are shown in figure 1.4. Because of the small Moliere radius of tungsten $(9 \mathrm{~mm})$, electro-magnetic showers are well contained even for such small calorimeters. In addition the incident position provided by the position sensitive layers is used to correct for shower-leakage.

Each plastic scintillator is viewed by an acrylic light guide and then read out by a PMT (HAMAMATSU R7400U) through $1 \mathrm{~mm}$ diameter optical fibers. The signals from the PMTs are amplified by pre-amplifiers installed on the top of the TAN (amplification factor $=4.8$ ) and sent to the counting room (USA15) through $200 \mathrm{~m}$ coaxial cables. Because of the light guide geometry, scintillation light is not collected uniformly over the area of a scintillator. The position dependence of light yield has been measured for each scintillator light-guide combination before detector assembly. The light yield from the scintillators was measured on a $4 \mathrm{~mm} \times 4 \mathrm{~mm}$ grid with a ${ }^{90} \mathrm{Sr}$ $\beta$-ray source through a $2 \mathrm{~mm}$ diameter collimator. An example of the measured non-uniformity of light yield is shown in figure 2.5. The correction of this non-uniformity was also checked with muons in a test beam at the SPS. Typical non-uniformity is about $\pm 10 \%$, and is corrected in further analysis as described in section 4.2.1.

The coaxial cable of $50 \Omega$ impedance (C-50-3-1) has a delay of $4.23 \mathrm{~ns} / \mathrm{m}$ and attenuates the pulse height from the amplifiers by a factor of 5 but transfers $90 \%$ of the total charge. To obtain linearity over a wide dynamic range, the combination of scintillator and PMT have been carefully chosen. The scintillator has a long decay time $(9.4 \mathrm{nsec})$ to reduce the peak current in the PMT. Also the bias resistor network of the PMT was optimized to assure a good linear response over a wide dynamic range. 

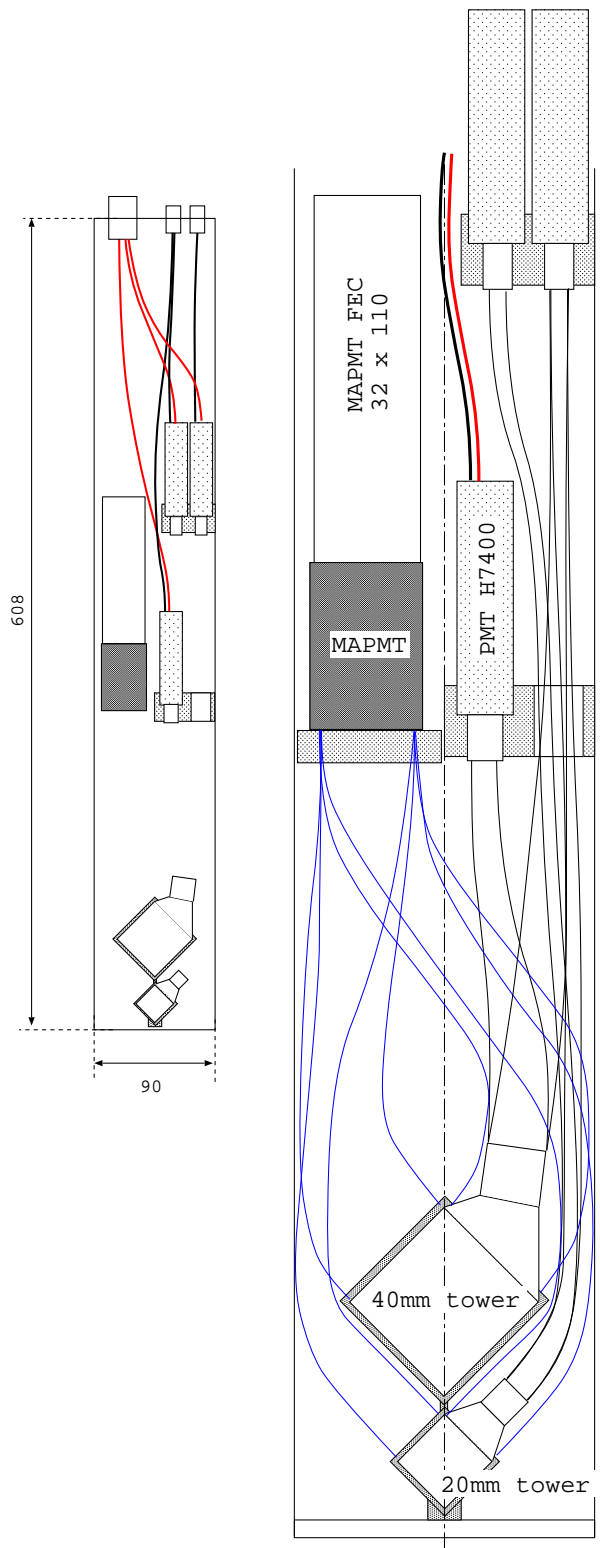

Figure 2.2: Front cut-away view of detector 1 showing the structure of the calorimeters, flexible light guide fibers, PMTs, MAPMTs front-end circuit box and inner cabling. The right figure is an enlarged view of the left one.

The linearity of the system is measured using a fast nitrogen laser (USHO KEN-1020; emission wavelength at $337 \mathrm{~nm}$ ). Because the nitrogen laser directly excites the wavelength shifter in the scintillator with a $0.3 \mathrm{nsec}$ pulse, the time profile of the scintillation light is nearly the same as that excited by a charged particle. Figure 2.6 shows the typical linearity of the PMT response with different bias HV. The modified PMT shows good linearity with a typical HV of $400 \mathrm{~V}$ while a single minimum ionizing particle (MIP) can be detected with about $1000 \mathrm{~V} \mathrm{HV}$.

These linearity curves have been measured for all PMT's for various values of bias HV. At typical operating HV, the linearity was checked for all the PMT's by changing the intensity of the 

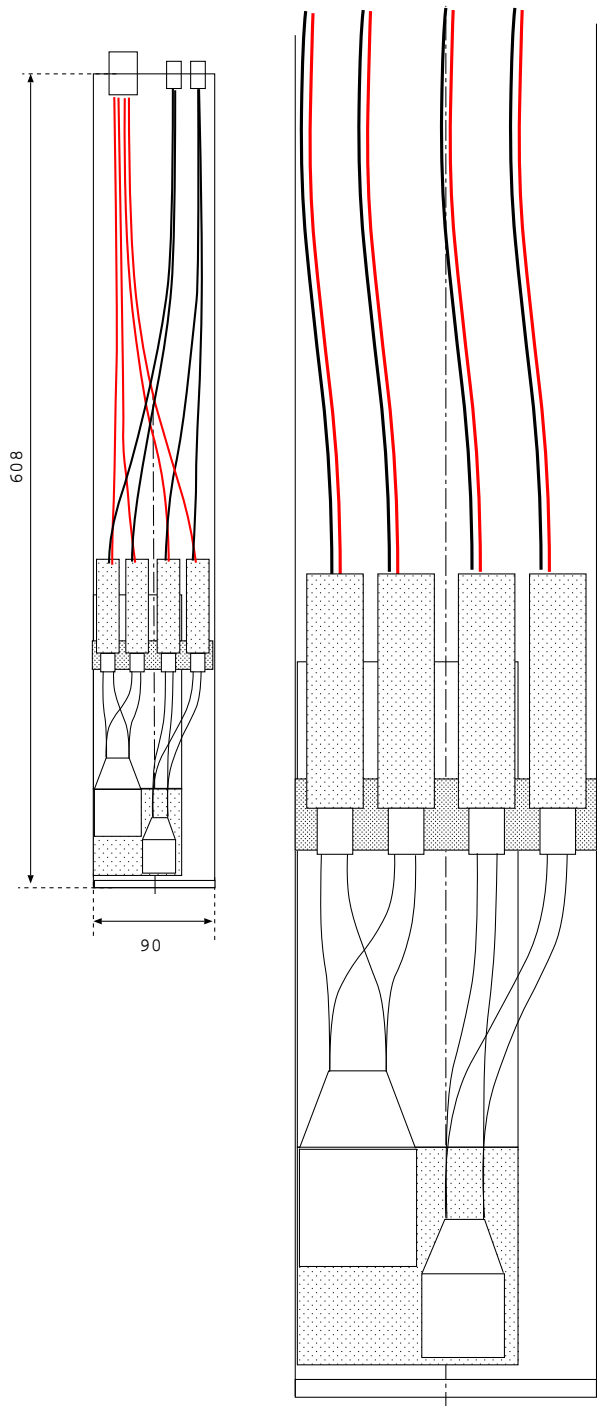

Figure 2.3: Front cut-away view of detector 2 showing the structure of the calorimeters, flexible light guide fibers, PMTs, silicon layers, their front-end circuit boards and inner cabling. The right figure is an enlarged view of the left one.

fast nitrogen laser precisely with a variety of neutral density (ND) filters. Figure 2.7 shows an example of the linearity of the PMT response. Here the horizontal axis indicates the nitrogen laser intensity in units of the equivalent number of shower particles (MIPs) detected at a layer. The vertical axis is the output of the PMT. Thus all the PMTs were checked to assure good linearity $\left(<5 \%\right.$ deviation) for incident light intensity varying from that corresponding to 1 MIP up to the $10^{5}$ MIPs expected at the shower maximum of a $10 \mathrm{TeV} \gamma$-ray.

In order to monitor and correct for time variation of gain or radiation damage to the scintillators, a fast nitrogen laser will be installed in USA15 for periodically illuminating each scintillator through $200 \mathrm{~m}$ bundles of quartz fibers. Pulse to pulse variation of laser intensity is about $20 \%$ and will be monitored during the run by two dedicated PMTs at USA15. 

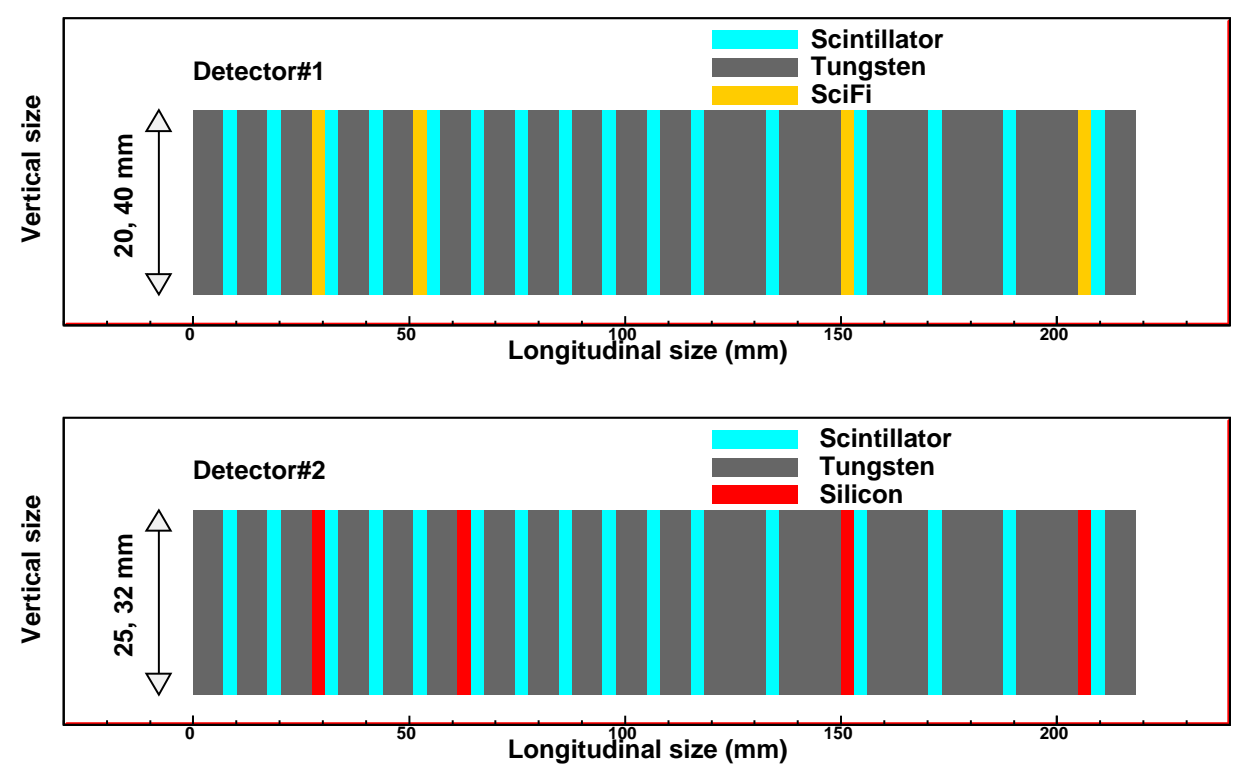

Figure 2.4: Longitudinal structure of detector 1 (top) and detector 2 (bottom); grey, light blue, orange and red indicate the layers of tungsten, plastic scintillator, SciFi and silicon strip sensor, respectively.

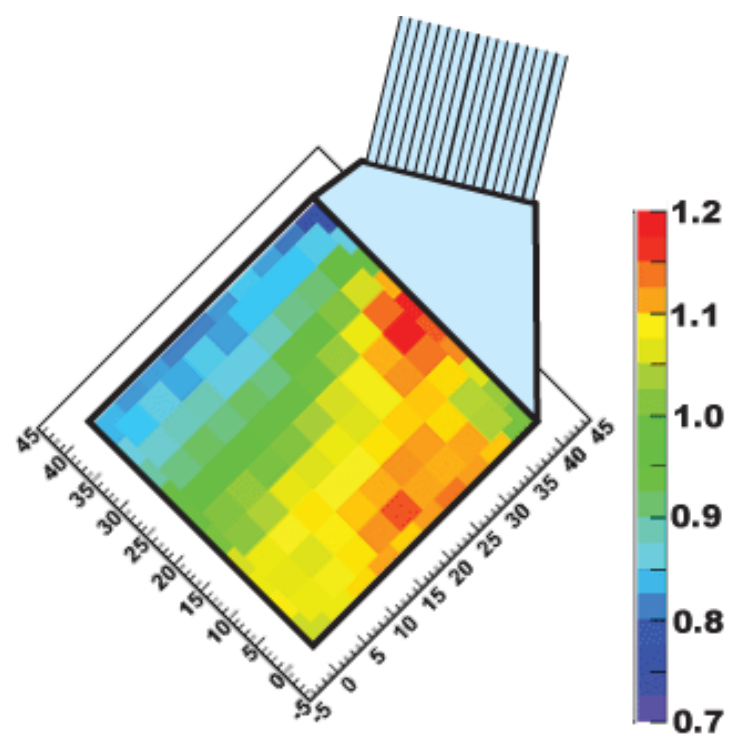

Figure 2.5: Position dependence of the light yield in a plastic scintillator. Color scale indicates relative signal intensity generated by $\beta$-rays.

\subsection{SciFi and MAPMT}

The position sensor of detector 1 is composed of 4 X-Y pairs of SciFi belts (KURARAY SCSF38). A SciFi belt consists of 20 (40) SciFi's in a single hodoscope plane for the $20 \mathrm{~mm}(40 \mathrm{~mm})$ 


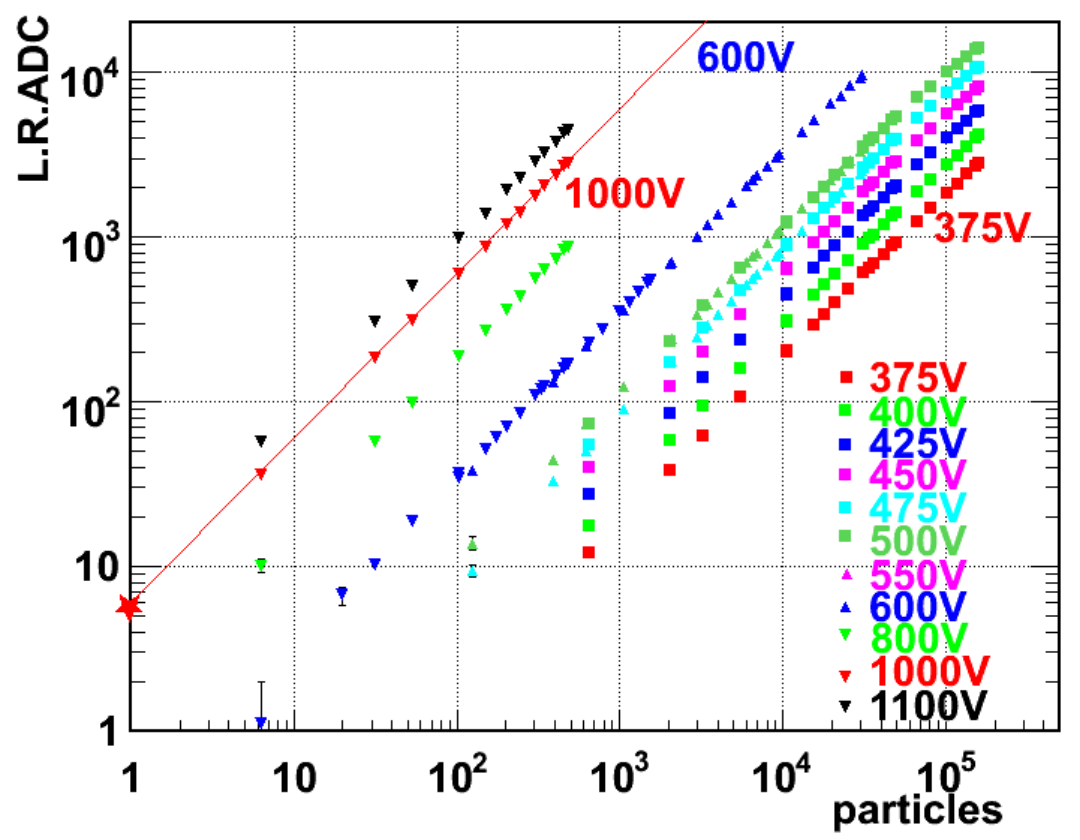

Figure 2.6: Linearity of scintillator and PMT excited by a nitrogen laser. Horizontal axis is the incident laser intensity in units of equivalent MIPs and vertical axis is AD count of the PMT output ( 1 AD count corresponds to $0.025 \mathrm{pC}$ ). Results are given for PMT HV bias from $375 \mathrm{~V}$ to $1100 \mathrm{~V}$.

calorimeter. Each SciFi has $1 \mathrm{~mm}$ square cross-section. The SciFi belts are painted with white acrylic water paint to improve light collection efficiency, to reduce cross talk between SciFi's, and to fix the SciFi's in a plane. Because fixing the SciFi's with paint is not very strong, both surfaces of a SciFi belt are covered with kapton tape. Hodoscope planes of the $20 \mathrm{~mm}$ and 40mm calorimeters that are inserted in the same layer are glued on the acrylic frame (figure 2.8) and pinned together with the scintillator and tungsten layers with G10 rods. The total number of SciFi channels is 480. Each SciFi is connected channel by channel with optical cement to a clear round fiber of $1 \mathrm{~mm}$ diameter. The clear fiber is attached as a light guide to one of 64 anodes of a MAPMT (HAMAMATSU H7546). The SciFi photon yield of a single MIP corresponds to more than 5 photo electrons. We need 8 MAPMTs to read out 480 channels of SciFi's, so the total number of channels of the 8 MAPMTs (512) exceeds that of the SciFi's.

\subsection{Silicon strip detector}

The position sensor of detector 2 is composed of 4 planes of microstrip silicon sensors. Each plane consists of 2 single-sided sensors, identical to those used by the ATLAS experiment for the barrel part of the Semi-Conductor Tracker (SCT) [13], which are used for the measurement of two orthogonal coordinates. These silicon sensors have $64 \mathrm{~mm} \times 64 \mathrm{~mm}$ total surface area, which is enough to cover the entire cross section of the calorimeter, and are produced on a $285 \mu \mathrm{m}$ thick n-type wafer. A sequence of $768 \mathrm{p}+$ microstrips with $80 \mu \mathrm{m}$ pitch is implanted on the junction side. The PACE3 chip, a 32 ch device designed to work with the LHC $40 \mathrm{MHz}$ clock and produced for the 

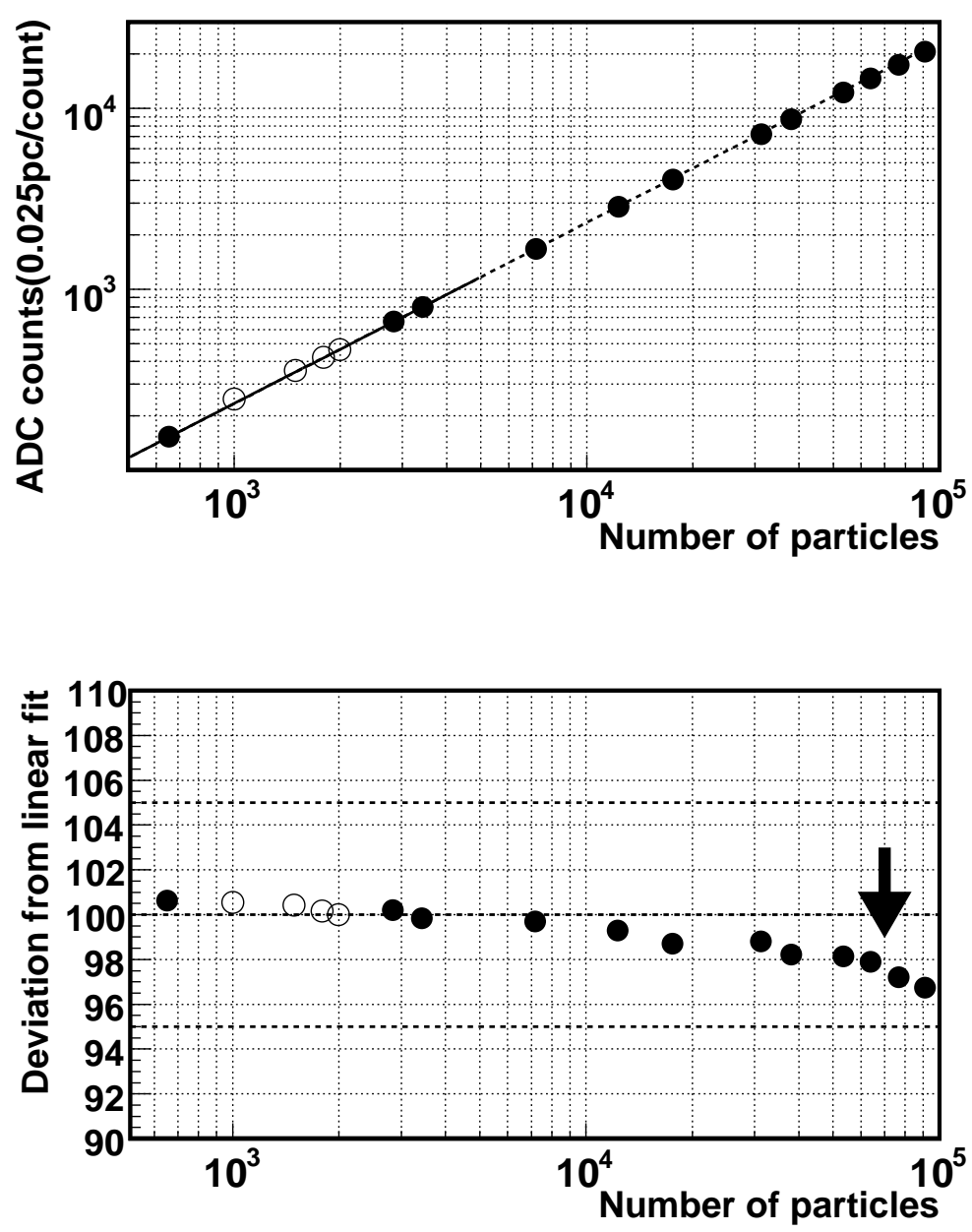

Figure 2.7: Linearity and dynamic range for one of the PMTs at nominal HV. The filled circles show the result of the laser calibration while the open circles show the result of the beam test at SPS. Horizontal axis shows relative intensity of the laser light in units of the equivalent number of MIPs determined from the beam test. The mean number of shower particles at the $7 \mathrm{TeV} \gamma$-ray shower maximum is indicated by an arrow.

CMS Silicon Preshower Detector [14], has been chosen for the front-end read-out. With a properly adjusted working point, the chips have a high dynamic range (up to 600 MIPs with a maximum deviation from linearity of $6 \%$ ). Due to the limited space inside the TAN and the small Moliere radius in tungsten, we decided to read out every other strip; the read-out pitch is hence $160 \mu \mathrm{m}$. In this way we have a total of 384 channels for each silicon sensor (12 PACE3 chips, housed on two printed circuit boards). The silicon sensor is glued on a thin fiberglass fan-out circuit that is also used to provide bias to the sensor through its backplane by means of a conducting glue [15]. Microstrips on the silicon sensors have been wire-bonded to the fan-out lines in the clean room of INFN-Florence. The front-end electronics are glued to dedicated kapton fan-out circuits, that in turn are wire-bonded to the boards and to the fiberglass circuit, as shown in figure 2.9. Each tracking layer is then glued on a $0.5 \mathrm{~mm}$ thick aluminum layer (used for mechanical support) and 


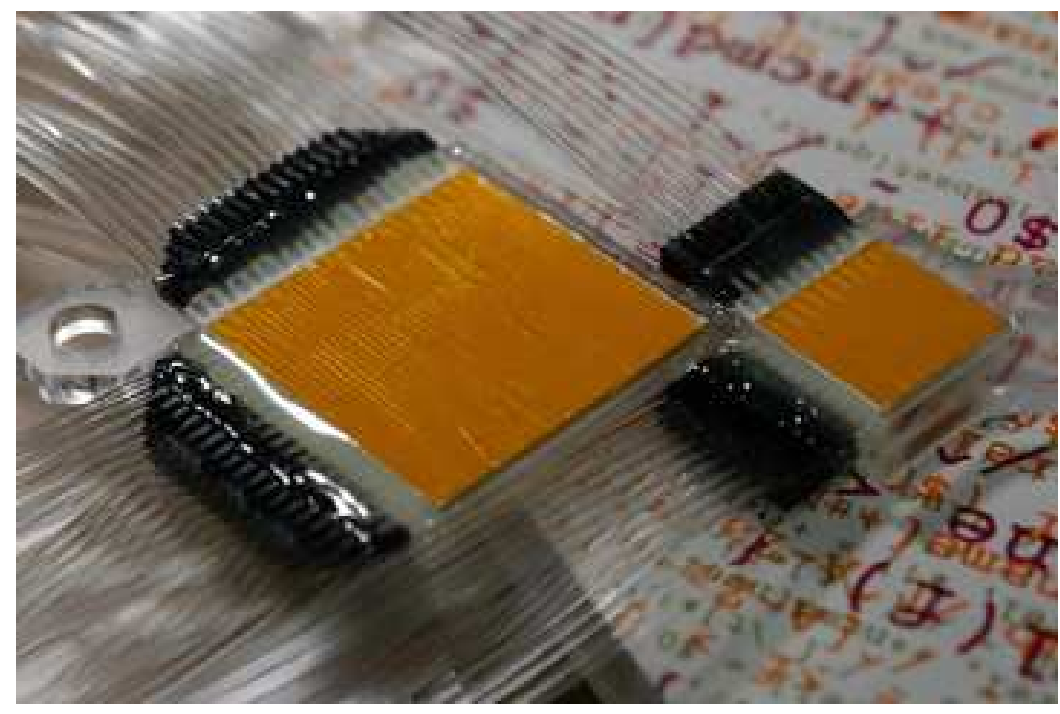

Figure 2.8: Photo of the SciFi hodoscope glued on the acrylic frame. Left and right parts are single hodoscope planes of $40 \mathrm{~mm}$ and $20 \mathrm{~mm}$ calorimeters, respectively.

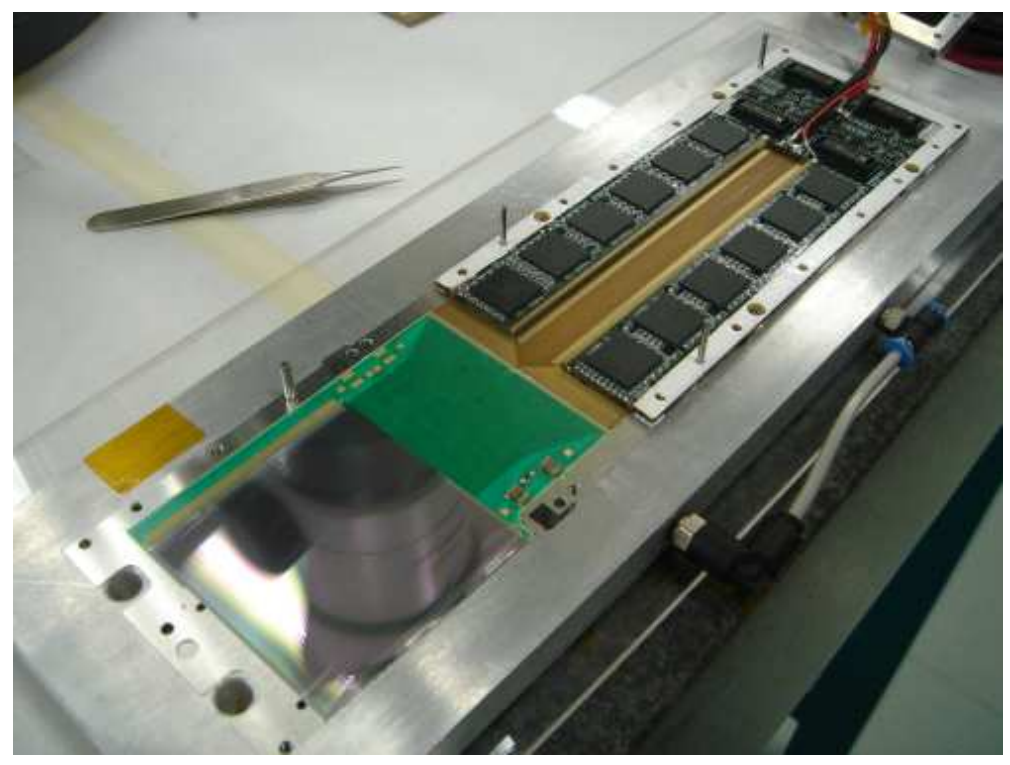

Figure 2.9: Photograph of a silicon layer during the assembly phase.

covered by a black Delrin frame on the silicon and by a $8.5 \mathrm{~mm}$ thick aluminum frame on the circuitry part. This metal frame is also used to extract the heat produced by the chips (silicon frontend circuits dissipate approximately $60 \mathrm{~W}$ in total) by means of a good thermal contact with the thick copper wall used in the detector package of detector 2 . 


\subsection{Manipulator}

The manipulators move the detectors in the vertical direction in order to increase the range of $\mathrm{P}_{T}$ coverage as well as to retract the detectors from the beam line to avoid unnecessary radiation damage when data is not being taken. The manipulators can be operated remotely. Considering the limited access to the TAN area and its high radiation environment, the manipulators were designed to be as robust as possible to avoid any mechanical trouble during LHC operation. A DC motor is driven by DC power supplied from the control room. The movement direction is determined by the polarity of the power supply that is also switched in the control room. Two mechanical switches are installed at the top and bottom to limit the stroke.

The absolute position of the detector is measured by two methods. One is to use a linear optical encoder (Mutoh DS-25) with a precision of $25 \mu \mathrm{m}$. When the motor is driven with $15 \mathrm{~V} \mathrm{DC}$ nominal voltage, the detector moves with a speed of $10 \mathrm{~mm} / \mathrm{min}$. During movement the encoder sends out two sequences of pulses with different phases. A counter installed in the counting room receives these pulses and gives the position of the detector. However, generally, optical encoders are known to be susceptible to radiation damage. Consequently a potentiometer has been prepared for a backup. The potentiometer (Mutoh RECTI P12) is simply a variable resistance with the sliding point moving with the detector. By measuring two variable resistances $\left(\mathrm{R}_{1}\right.$ and $\left.\mathrm{R}_{2}\right)$ and calculating $P_{\text {pot }}=\frac{R_{1}}{R_{1}+R_{2}}$, one can obtain a good position resolution. The use of a resistance ratio compensates for the possible effect of temperature change. To determine the resolution we compared the optical encoder value and $P_{\mathrm{pot}}$ measured at the same time for various positions. The rms deviation of the residual from a linear fit was $70 \mu \mathrm{m}$. This same result was obtained several times at intervals of one week.

\subsection{Front counters}

The FCs have a thickness of $8 \mathrm{~mm}$ and are inserted in front of detector 1 and detector 2 . Two pairs of thin plastic scintillators ( $40 \mathrm{~mm} \times 80 \mathrm{~mm} \times 2.0 \mathrm{~mm}$; Saint-Gobain Crystals BC404) are aligned in the vertical and horizontal directions to compose a double layer counter as shown in figure 2.10. Between the two layers, a copper plate of $0.5 \mathrm{~mm}$ thickness is inserted. Scintillation light outputs are sent through an acrylic light guide and optical fibers to four PMTs (HAMAMATSU H316410) placed outside of the TAN instrumentation slot. The PMT signals are sent to the counting room and recorded by ADCs. The signals are also sent to discriminators and may be used for an additional trigger information. The FC can be used to tag IP interactions with particles produced in both Arms with as wide an acceptance as possible for reducing background events produced by beam-gas interactions and beam-halo interactions with the beam pipe upstream of the TAN. The FC can also be used in anti-coincidence mode to ensure that neutral particles are being detected by the calorimeters and not charged particle background produced by beam-gas and beam-halo interactions. Using the segmented structure of the FC, we also expect a certain level of position measurement of incident particles. 


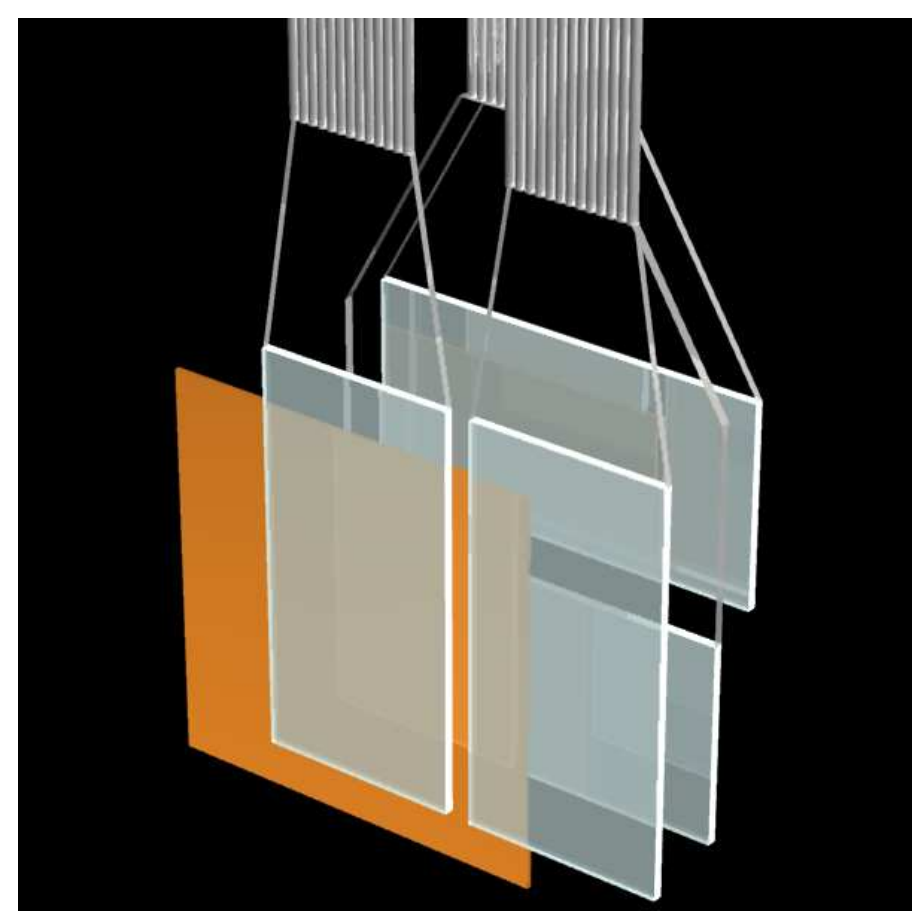

Figure 2.10: A schematic view of a front counter (FC). Two pairs of plastic scintillators segmented in vertical and horizontal directions are indicated by light blue rectangles. A copper plate of $0.5 \mathrm{~mm}$ thickness is inserted between the pairs. The scintillator light outputs are fed to PMTs via an acrylic light guide and optical fibers. 


\section{Chapter 3}

\section{Trigger and DAQ electronics}

\subsection{Global flow of trigger and DAQ}

Data acquisition is carried out in the ATLAS counting room (USA15). All the sequences are optimized for 43 bunch operation. A timing diagram of trigger and data acquisition is shown in figure 3.1. Signals from the detectors reach the electronics in the counting room though $200 \mathrm{~m}$ cables. A first level trigger is generated when a bunch crossing (BX) occurs that can be identified using two BPTX signals generated by two Beam Position Monitors (BPMs) installed $175 \mathrm{~m}$ away from the interaction point. At every first level trigger, a $500 \mathrm{nsec}$ gate signal is sent to the ADC for the plastic scintillators (CAEN V965). The particle flight time from the interaction point to our detectors is $466 \mathrm{nsec}$ and the signal propagation time between the detector and USA15 is $860 \mathrm{nsec}$. The signals from plastic scintillators arriving at USA15 are split into ADCs and discriminators (CAEN V814B) by custom made NIM FANOUT modules with amplification factors of 1 and 4, respectively. The outputs of the discriminators are sent to VME FPGA boards (GND GPIO GN-

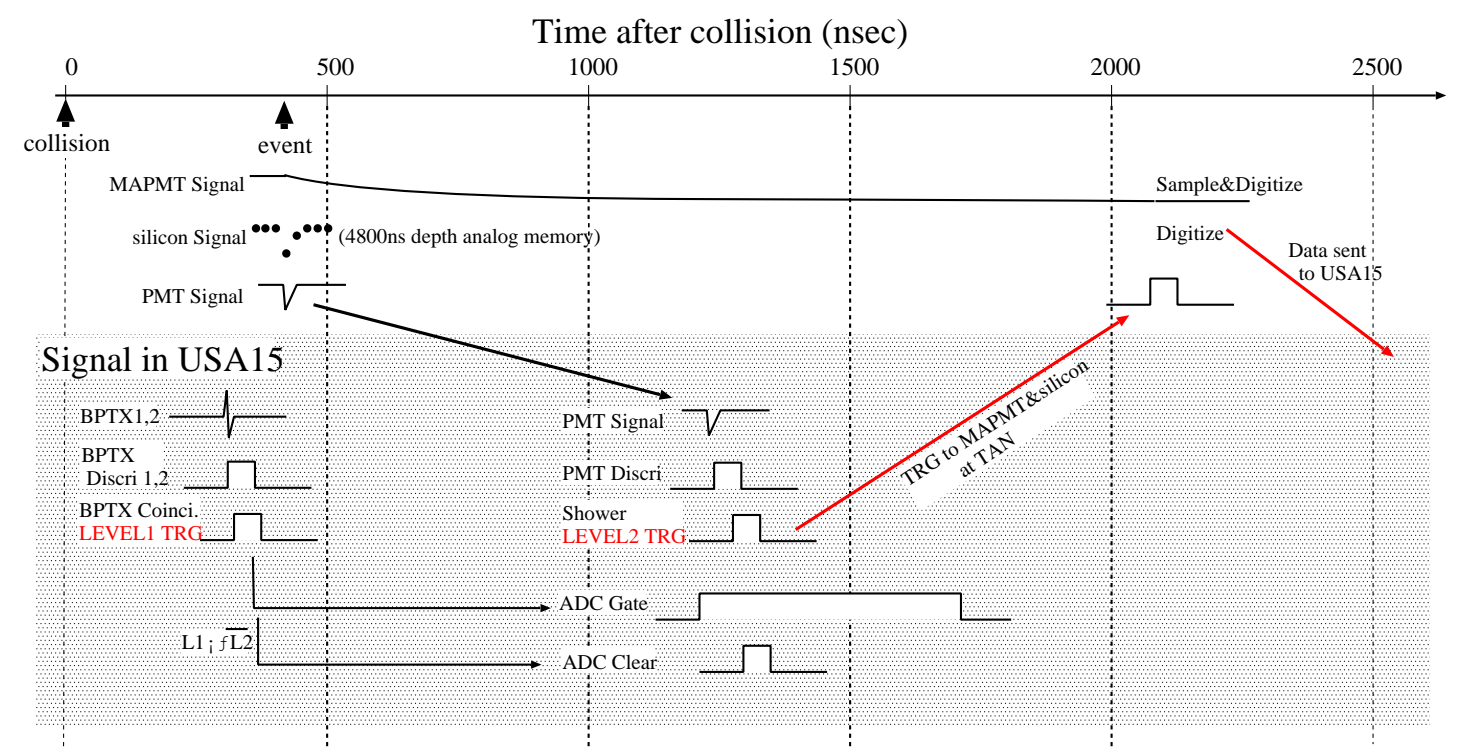

Figure 3.1: The timing chart for trigger and DAQ. 
0324-3). Then the second level trigger is issued when more than 3 successive layers detect more than 300 particles corresponding to the energy threshold of about $100 \mathrm{GeV}$. Details of the trigger logic and energy threshold will be further optimized considering the actual beam conditions at the time of LHC operation. If the second level trigger is not generated within $1 \mu \mathrm{sec}$ after the first level trigger, the $\mathrm{ADC}$ is cleared. At the second level trigger, if the acquisition $\mathrm{PC}$ is enabled to read data, a final signal to collect all the data is issued. For the SciFi in detector 1, the second level trigger is sent to the FECs to sample and digitize the MAPMT signal as described in section 3.2. For the silicon tracker in detector 2, the signal stored in the analog memory at the proper timing is digitized as described in section 3.3. Then digitized pulse height data is sent to the counting room and recorded together with the calorimeter data.

The data acquisition is carried out independently for the two arms. However in case of a high background counting rate, which can be expected if the residual gas in the beam pipe is far worse than expected, we can use a coincidence between the two arms to suppress this background. The FC with large acceptance coverage are expected to supply coincidence signals with the detector on the opposite side.

The data acquisition is carried out using the MIDAS package (Maximum Integration Data Acquisition System, [16]). One MIDAS server PC controls two front end PCs for detector 1 and detector 2. The raw data sizes of a single event for two arms are 1.2 kbytes and 14 kbytes in the MIDAS format. The MIDAS server PC also performs a RAID1 storage and can store up to 1.5 Tbytes of data, corresponding to about $10^{8}$ events from both arms. Two other PCs, one for each arm, also communicate with the MIDAS server for a fast analysis during the run. The MIDAS format can be converted to the ROOT format by which fast on-line analysis will be made with the standard ROOT analysis tools [17]. Some basic results of the fast analysis (counting rate, event position, etc.) are distributed in the CERN network every second through the Data Interchange Protocol (DIP). Slow control data (electronics temperature, HV, LV values, manipulator position, etc) are also recorded. Some important machine conditions (luminosity, crossing angle, etc.) are also received through the DIP and recorded in our data base.

In order to synchronize with ATLAS events in possible further analysis, we count the number of the ATLAS level-1 (L1A) triggers and $40 \mathrm{MHz}$ clock (Bunch Clock or BC) counts. These counts are reset roughly every second by the Event Counter Reset (ECR) signal that is also issued from ATLAS. These counts are latched and stored in FIFO when a L1A signal arrives and readout when a LHCf trigger is generated. Time synchronization of the acquisition PC with the CERN time server with one second accuracy can enable us to correlate LHCf and ATLAS events. The combination of the PC clock and the BC count gives the absolute time of each event with a precision of $25 \mathrm{nsec}$. Because the interval between bunch crossings will be $2 \mu$ sec during LHCf operation, we can easily identify the correlated events. These methods will also be used to identify the coincident events of detector 1 and detector 2 .

\subsection{Electronics and DAQ for the SciFi detector}

The MAPMT signals are read out with the Front End Circuits (FECs) packed in the detector on which analog ASICs (VA32HDR14), ADCs and sequencers are mounted. Two VA32HDR14 chips, each of which contains 32 sets of preamplifier, shaping amplifier, sample and hold circuits, 
and one analog multiplexer, are used to read out the 64 channels for one MAPMT. Peaking time of the shaping amplifier is $1.9 \mu \mathrm{s}$. The peak voltages recorded by the sample and hold circuits are read out through an analog multiplexer. One ADC coupled to one VA32HDR14 performs the digital conversion of 32 channels in $64 \mu \mathrm{s}$. The digital data are transferred in a serial line from the detector via LVDS to an interface board installed on the VME bus. The processes after sampling are carried out when a trigger signal arrives at the FEC according to the level-2 trigger described in section 3.1. The trigger signal arriving $1.9 \mu \mathrm{s}$ after the shower matches with the peaking time of the shaping amplifier.

\subsection{Electronics and DAQ for the silicon tracking detector .}

The DAQ used for the silicon part of detector 2 is mainly based on the fast electronics developed for the large LHC experiments, inserted in custom made boards and produced taking into account the LHCf requirements.

The analog preamplifier (PACE3) outputs are digitized by custom made ADC boards with 12 bit resolution. These boards are mounted, piggy-back, on a mother board which also takes care of the optical link communications and clock, level-2 trigger and slow control distribution to the PACE3 chips. Two silicon detectors are serviced by one mother board in such a way that a total of 4 mother boards are installed in the TAN area. The digitized data, along with other status information, are sent via optical fiber links to a VME receiver board in the Atlas USA15 counting room. The receiver board formats the data in an appropriate way for the common detector $2 \mathrm{DAQ}$ to digest. The PACE3 chips receive the LHC $40 \mathrm{MHz}$ clock and level-2 trigger through the TTCrx system developed at CERN for LHC [18]. The signals are coded through a Front End Controller (FEC) module (developed for the CMS experiment [19]) which also takes care of the I2C commands needed to properly set the PACE3 internal registers. From the FEC these fast control signals are sent through optical links down to the experiment in the tunnel. A custom receiver board then decodes the signals and sends them via an electrical token ring to the Clock and Control Unit Modules(CCUM) on the mother boards. These CCUM [19], developed for the CMS tracker, decode both clock, trigger and I2C commands for the PACE3 chips. 


\section{Chapter 4}

\section{Performance of the LHCf detectors}

\subsection{Expected performance of the LHCf detectors}

Here we present the results of Monte Carlo studies for evaluating the performance of the LHCf detectors described in section 2. Detector performance was also checked by beam tests described later in section 4.2 In these studies, EPICS [20], which is widely used in air shower experiments, was used for detector simulation. EPICS simulates all electromagnetic and hadronic processes which have been well benchmarked by results from EGS4 or CERN test beam experiments. The primary hadronic interaction model used in this section is DPMJET3 [21] which is also commonly used in air shower simulations.

\section{Energy resolution}

The LHCf detectors consist of tungsten sampling shower calorimeters ( $44 X_{0}$ and $\left.1.7 \lambda\right)$ with compact apertures which results in shower particle leakage from the edges of the calorimeters.

The number of shower particles contained in calorimeter is plotted in figure 4.1 as a function of the distance between the shower center and the nearest edge. Though the leakage fraction is not negligible, we have found that it does not depend on the energy of the incident primary particle. Using the shower position measured by the position sensitive layers together with the leakage function in figure 4.1, the measured shower energy can be corrected for shower leakage.

The expected energy resolution of the $20 \mathrm{~mm} \times 20 \mathrm{~mm}$ calorimeter after shower leakage correction is plotted in figure 4.2 as a function of incident $\gamma$-ray energy. Here the $\gamma$-ray energy is reconstructed from the energy deposited by shower particles after the 3rd tungsten layer (at $6 X_{0}$ ) in order to prevent contamination due to background of low energy $\gamma$-rays. Selecting events with shower axis $2 \mathrm{~mm}$ or more from the nearest edge of the calorimeter and applying the leakage correction, the expected energy resolution is $6.3 \%$ for $100 \mathrm{GeV}$ and $2.8 \%$ for $1 \mathrm{TeV} \gamma$-rays.

While the total length of the calorimeters expressed in nuclear interaction lengths is small $(1.7 \lambda)$, the detector still can be used as a hadron shower calorimeter. To minimize longitudinal shower leakage, events are selected which start their shower development near the front of the detector. Selecting events for which the shower axis is $2 \mathrm{~mm}$ or more from the closest edge, and requiring that the energy deposited in the first radiation length exceeds that deposited by 20 MIPs, 

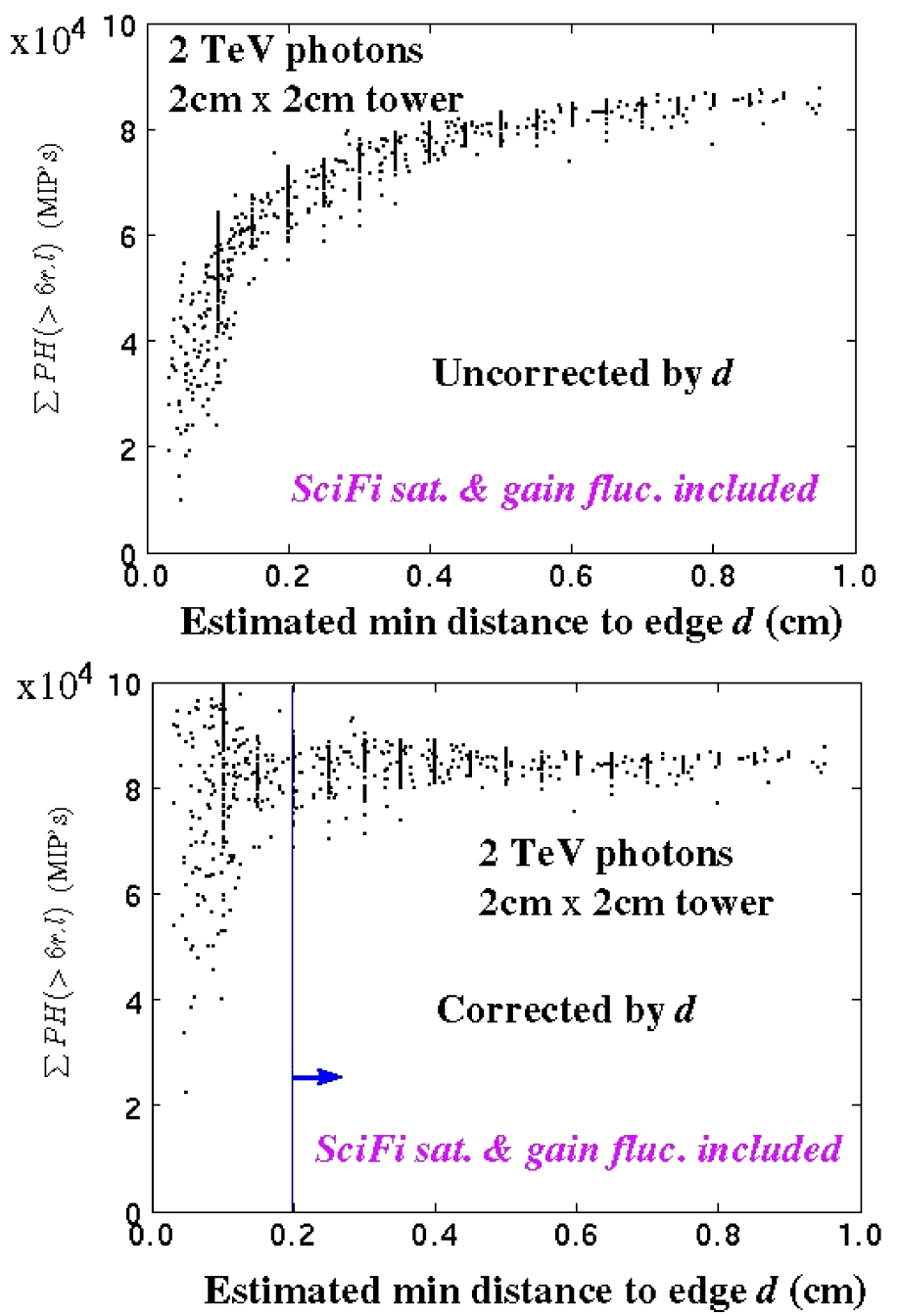

Figure 4.1: Example of the shower leakage function for $2 \mathrm{TeV} \gamma$-rays for the $20 \mathrm{~mm} \times 20 \mathrm{~mm}$ calorimeter. The upper plot shows the number of shower particles after $6 \mathrm{X}_{0}$ as a function of distance of the incident position from the nearest edge. The lower plot shows the same data after correction by the leakage function. The blue arrow shows the fiducial area where the incident shower positions are $2 \mathrm{~mm}$ or more from the nearest edge.

then a $30 \%$ energy resolution is expected for $6 \mathrm{TeV}$ neutrons. The detection efficiency after applying the cuts discussed above is about $4 \%$.

\section{Position determination}

The simulated position resolution of the SciFi layers for detector 1 is shown in figure 4.3. The incident shower position is estimated from the fiber with the peak signal and the adjacent 2 fibers weighted by their ADC counts. The resolution is better than $0.2 \mathrm{~mm}$ over the energy range of $100 \mathrm{GeV}$ to $7 \mathrm{TeV}$ though it worsens at higher energy due to saturation. The position resolution of the silicon layers of detector 2 was also simulated and it is $15 \mu \mathrm{m}$ for $1 \mathrm{TeV} \gamma$-rays. The effect of saturation is almost negligible at this energy. 

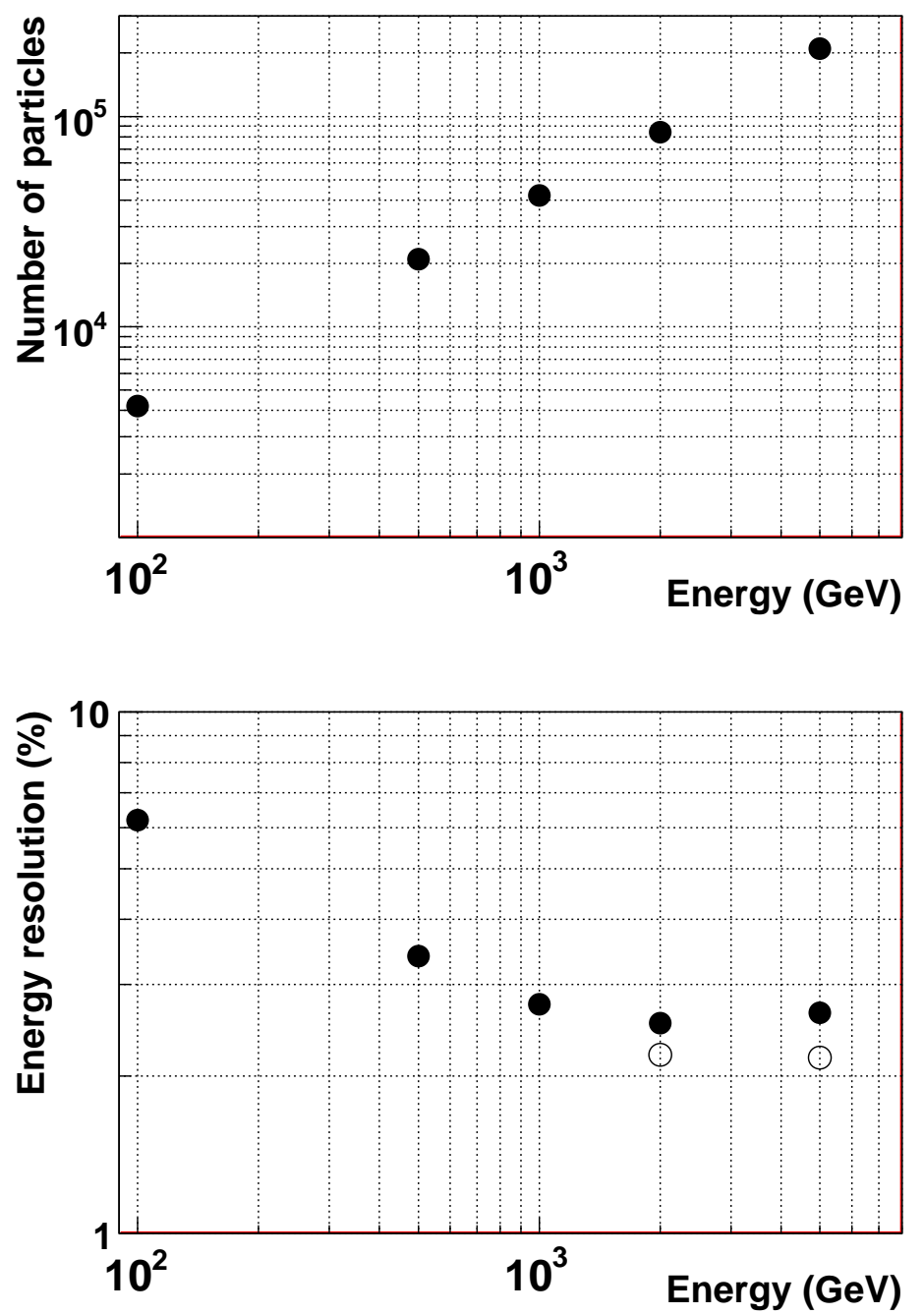

Figure 4.2: The linearity and resolution in the $\gamma$-ray energy determination. The upper plot shows number of shower particles after $6 \mathrm{X}_{0}$ and after the shower leakage correction. The lower plot shows energy resolution up to $7 \mathrm{TeV}$. Here the open circles are the data before and the filled circles after the saturation effect of the SciFi has been taken into account.

\section{Detector geometry and acceptance}

The LHCf detectors are installed between two beam pipes embedded in the TAN absorbers $\pm 140 \mathrm{~m}$ from the IP. At this location, the inner beam separation dipole has swept away all the charged secondary particles so that only neutral particles such as $\gamma$-rays from $\pi^{0}$ decays, neutrons or neutral kaons reach the detector. In the case of $7+7 \mathrm{TeV}$ collisions, their flux is mostly concentrated within a few $\mathrm{cm}$ around the center of the neutral particle flux arriving from the IP, which is the direction of the proton beam at collision projected to the detector plane.

Figure 4.4 shows the geometrical acceptances for single $\gamma$-ray events. Here the acceptance is drawn as a function of the distance between the center of the neutral particle flux and the impact point of a particle on LHCf. Without beam crossing angle, detector 1 will locate the center of 


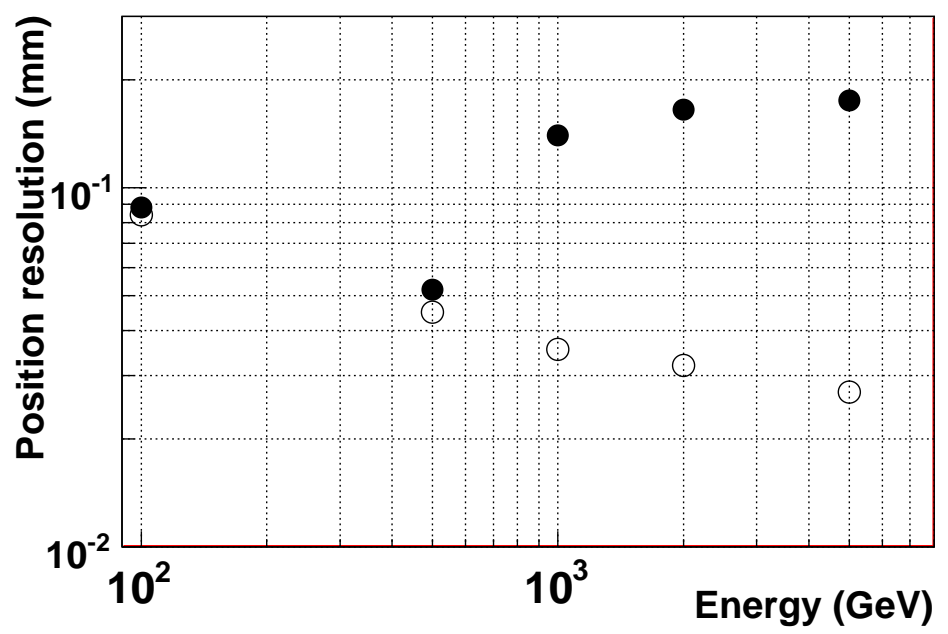

Figure 4.3: Position resolution of SciFi up to $7 \mathrm{TeV}$. The effect of saturation of the SciFi is taken into account in the filled circles but not in the open circles.

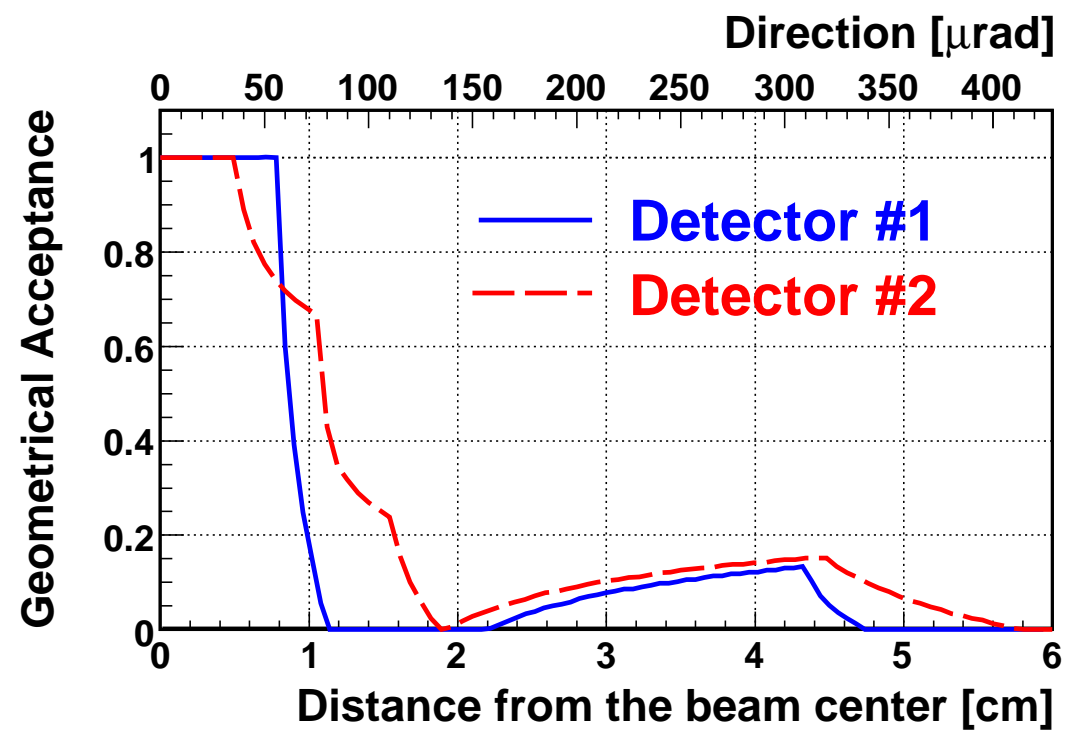

Figure 4.4: Geometrical acceptance of detector 1 (solid blue) and detector 2 (dotted red) for single $\gamma$-ray events as function of the distance from the beam axis.

$20 \mathrm{~mm} \times 20 \mathrm{~mm}$ calorimeter at the center of beam-pipe where center of the flux of neutral particles exists. In this case, about half of the $40 \mathrm{~mm} \times 40 \mathrm{~mm}$ calorimeter will be cut off by the beam-pipe aperture of $\pm 43.8 \mathrm{~mm}$ at the D1 magnet location. With a beam crossing angle of $140 \mu \mathrm{rad}$, the center of neutral particle flux moves downward by $2 \mathrm{~cm}$. In this case the center of $20 \mathrm{~mm} \times 20 \mathrm{~mm}$ calorimeter will be adjusted downward by $2 \mathrm{~cm}$ and the $\mathrm{P}_{T}$ region covered by the detector will be enhanced.

Figure 4.5 shows the $\mathrm{E}_{\gamma}-\mathrm{P}_{T \gamma}$ correlation plot of photons for $7+7 \mathrm{TeV}$ proton collisions. The curve shows the $P_{T}$ acceptance of detector 1 defined by the vertical aperture of the beam pipe in the D1 magnet. The upper and the lower curves correspond to the beam crossing angles of $140 \mu \mathrm{rad}$ 


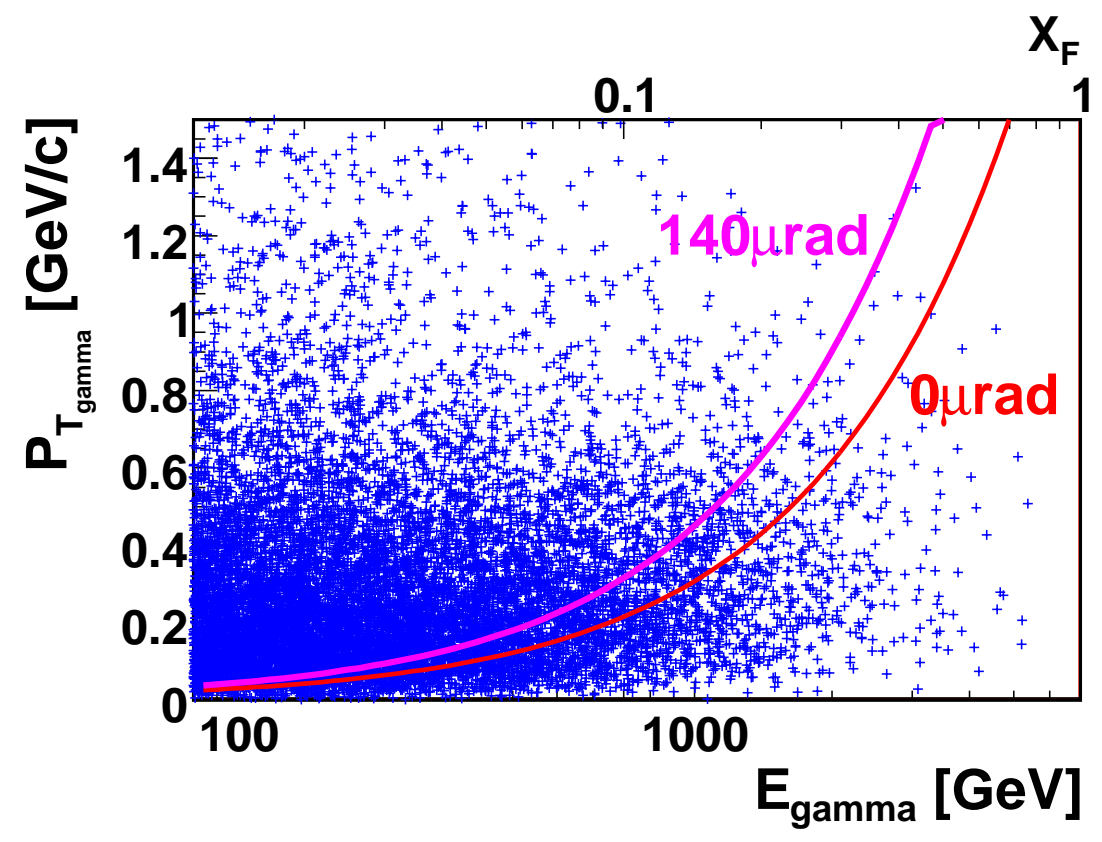

Figure 4.5: The $\mathrm{E}_{\gamma}-\mathrm{P}_{T \gamma}$ correlation plot. High energy photons with small $\mathrm{P}_{T_{\gamma}}$ can be recorded by the LHCf shower counter. The curves show geometrical cuts for our shower calorimeter arising from the configuration of the beam pipe and magnets. The upper curve (magenta) and the lower curve (red) correspond to beam crossing angles of $140 \mu \mathrm{rad}$. and $0 \mu \mathrm{rad}$, respectively.

and $0 \mu \mathrm{rad}$,respectively. The photons that fall in the area under the curves will be detected by LHCf. From these curves it can be seen that almost all $\gamma$-rays with energies higher than $2 \mathrm{TeV}$ can be detected by LHCf.

\section{$\pi^{0}$ reconstruction}

The $\pi^{0}$ mass can be reconstructed in the invariant mass distribution of two $\gamma$-rays, one each hitting the two tower calorimeters of detector 1 or detector 2 . The expected mass resolution is about $5 \%$ after taking into account $5 \%$ energy resolution and $0.2 \mathrm{~mm}$ position resolution.

The $\pi^{0}$ mass peak can be used for the absolute calibration of the energy scale. It also helps for rejection of beam-gas interactions by giving a constraint for location of the neutral pion production vertex. The acceptance of detector 1 and detector 2 for $\pi^{0}$ detection are shown in figure 4.6 for 1 , 2 and $5 \mathrm{TeV}$. In these estimations, the crossing angle is zero and the small calorimeters are located on the horizontal midplane as shown in figure 1.4. The lower horizontal axis gives the distance between the impact point defined by extrapolation of the $\pi^{0}$ trajectory at production to the detector and the center of the neutral particle flux from the IP. The top horizontal axis expresses this distance as the $\pi^{0}$ production angle. 

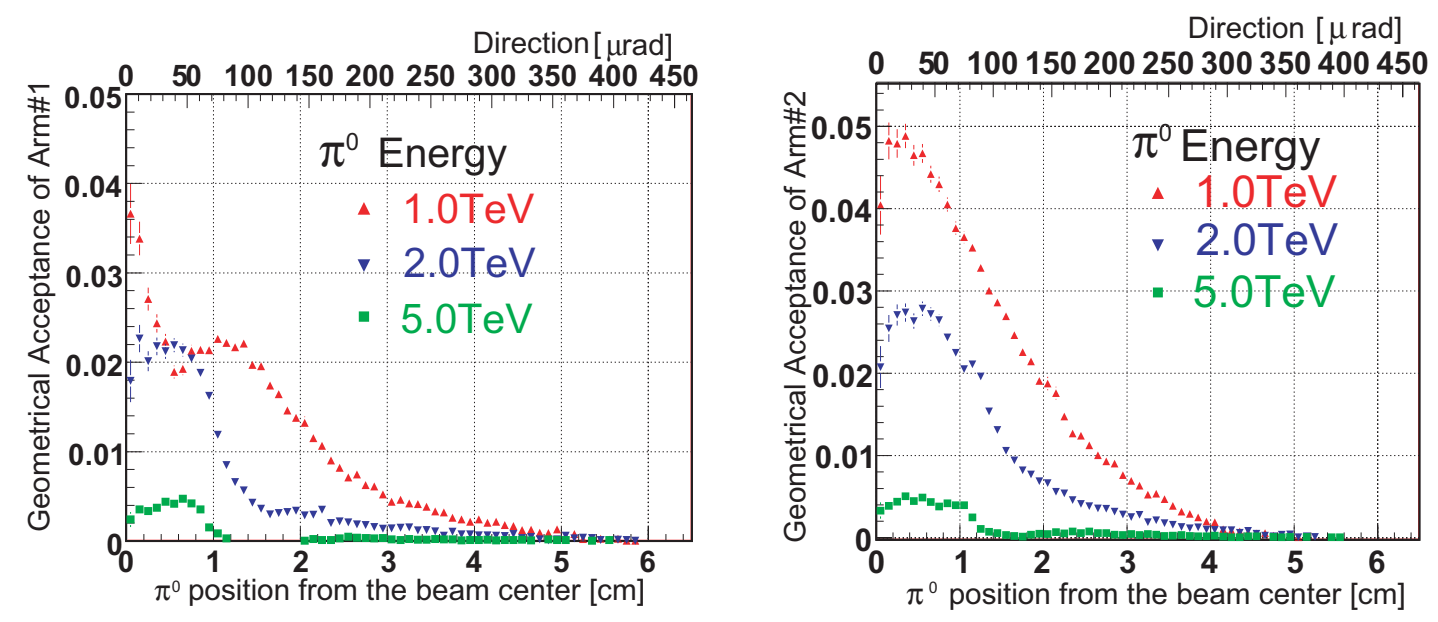

Figure 4.6: Geometrical acceptance for $\pi^{0}$ 's. Left and right plots show the acceptances for detector 1 and detector 2, respectively. Acceptances for three different $\pi^{0}$ energies are plotted as functions of the distance between the center of the neutral particle flux and the impact point defined by extrapolating the $\pi^{0}$ trajectory at production to the plane of the detector.

\subsection{Results of SPS beam tests}

The assembly of the detectors was finished in April 2007 and their performance was tested at the CERN SPS North Area H4 beamline from 24 August to 11 September 2007. Both detectors were exposed to electron, hadron and muon beams. Electron beams with energies of 50, 100, 150, 180 and $200 \mathrm{GeV}$, hadron beams with energies of 150 and $350 \mathrm{GeV}$ and a muon beam with an energy of $150 \mathrm{GeV}$ were used.

The beam test setup is illustrated in figure 4.7. One of the detectors was placed on a movable table in the beam area. Data from the calorimeters and position sensors was recorded when triggered by scintillators placed in front of the detector. At the same time, data from an external silicon strip detector (ADAMO) [22] installed between the detector and the trigger scintillators were also recorded. These data are used to precisely determine the incident position of the beam particle for comparison with the internal position sensitive layers.

In this article we briefly report preliminary results for detector 1 from the SPS2007 beam test. A detailed report on the analysis as well as results for detector 2 is in preparation.

\subsubsection{Results for the calorimeters}

In the beam test, the PMTs were operated at several different gains. Low gain is a default mode, in which PMTs were operated with a gain of 1000 . With this gain, the dynamic range of the calorimeters is optimized to cover $100 \mathrm{GeV}$ to $7 \mathrm{TeV}$ electromagnetic showers. Some higher gain modes ranging from 3000 to 10000 are optimized for measurements below $1 \mathrm{TeV}$ with improved resolution and possibly overcoming unexpected noise in the DAQ electronics. For the muon runs, which are only available in the SPS test beam, the PMTs were operated at the highest gain of about 


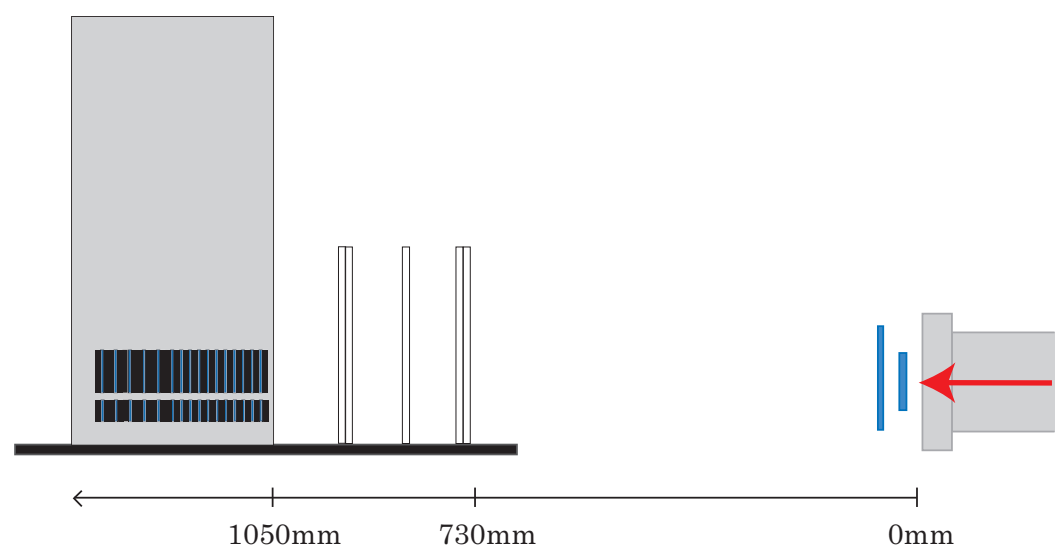

Figure 4.7: Setup of the SPS experiment. Beam enters from right along the red arrow. Two plastic scintillators indicated by blue rectangles were used to trigger beam particles. Five open rectangles show the silicon tracker, ADAMO, placed in front of the LHCf detector that is drawn as a big grey rectangle. The two black-blue structures shown in the detector are the calorimeters. The detector and ADAMO were placed on a movable stage.

$10^{5}$ to measure a single MIP. The relative gains of the different modes were measured during the pre-assembly calibration for each PMT as described in section 2.2.

The position of the shower axis was determined by the SciFi layers by finding the peak of the lateral shower shape. Gain calibration was carried out for all of the scintillating fibers in each SciFi layer. Position resolution was checked with ADAMO. The measured shower axis was used to correct for the position dependence of light yield from plastic scintillators and the shower leakage effect by referring to the previously measured non-uniformity maps and shower leakage studies.

The gain calibration of each plastic scintillator layer was carried out by using the electromagnetic showers produced by electron beams. Figure 4.8 shows an ADC distribution at the 4th layer for a $100 \mathrm{GeV} / \mathrm{c}$ electron beam. The energy that is expected from MC simulations of the shower particles is also shown. By comparing the two distributions a conversion factor from ADC to energy deposited by shower particles is determined for each layer. Conversion factors for different beam energies were obtained and are compared with each other in figure 4.10. We found these factors are consistent at the $2 \%$ level for $50-200 \mathrm{GeV}$ electrons. To check consistency with the energy deposited by a single MIP, the muon data was analyzed as shown in figure 4.9. In this comparison, the relative PMT gain difference for the different HV settings for the electron and the muon runs was taken into account. The pedestal fluctuations of $8 \mathrm{AD}$ counts (rms) due to electrical noise was also taken into account in the MC calculations. We can observe good agreement between the two distributions.

Summing up the signal in all the layers except the first, the energy resolution is defined as root-mean-square of the distribution. Here showers for which the incident particle position is reconstructed to lie $>2 \mathrm{~mm}$ from the nearest edge of the calorimeter are used. The energy resolutions obtained in the beam test and in MC simulation are plotted in figure 4.11 for high gain and low gain operation. Resolution was worst for $50 \mathrm{GeV}$ with low gain $(450 \mathrm{~V} \mathrm{HV})$ due to the electrical noise level during the SPS beam test. The electrical noise was negligible for the high gain mode 


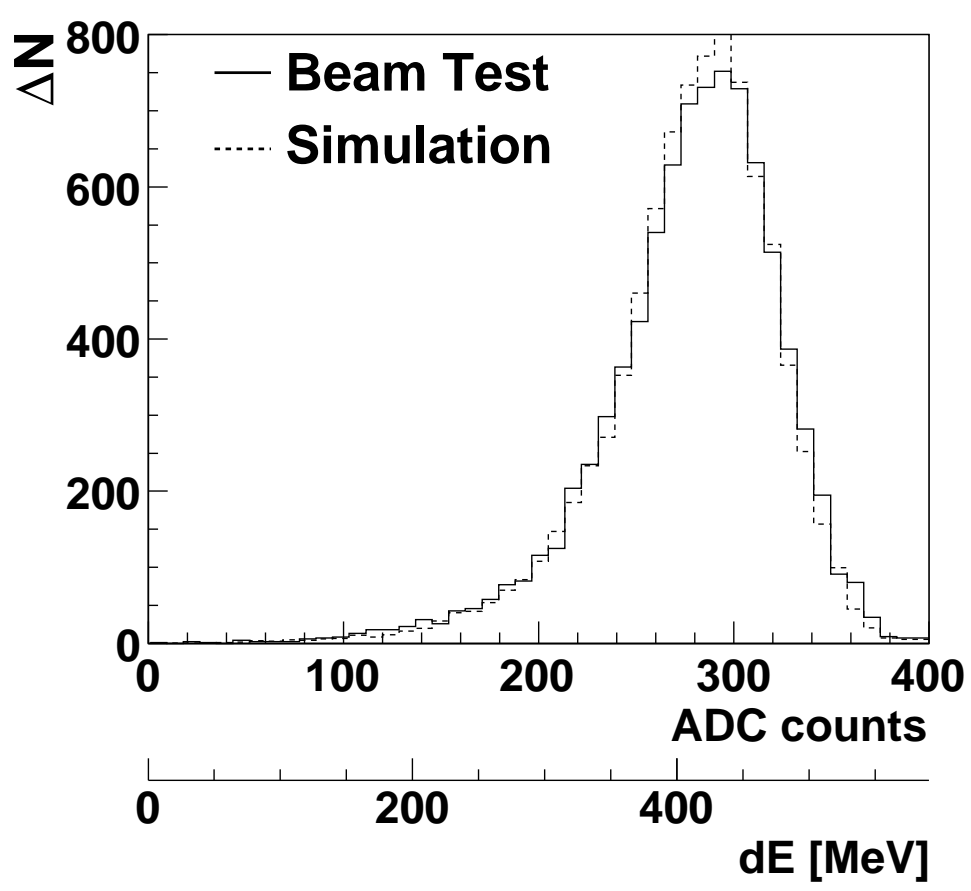

Figure 4.8: The ADC distribution at the 4th scintillation layer for $100 \mathrm{GeV}$ electron showers (solid). The distribution of deposited energy expected from MC calculations (dashed) is also shown.

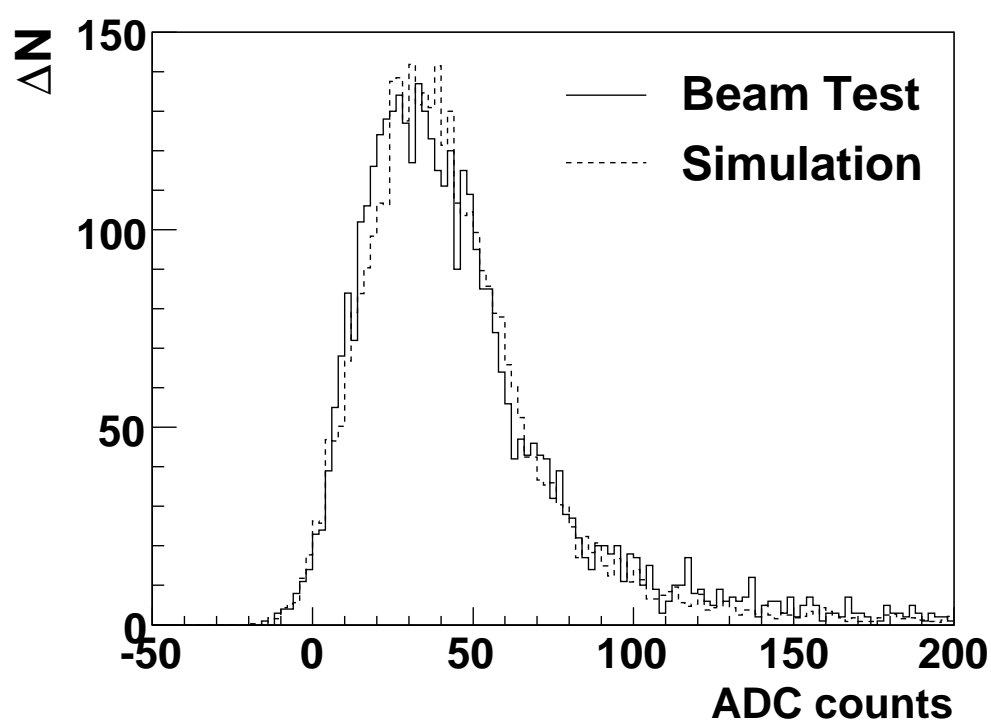

Figure 4.9: The $\mathrm{ADC}$ distribution at the 4-th scintillation layer for $150 \mathrm{GeV}$ muon tracks (solid). The energy distribution expected from MC calculations (dashed) is also shown.

(600V HV). In any case, we have obtained excellent agreement between the data and the MC simulations showing the energy resolution is satisfactory for the experiment. 


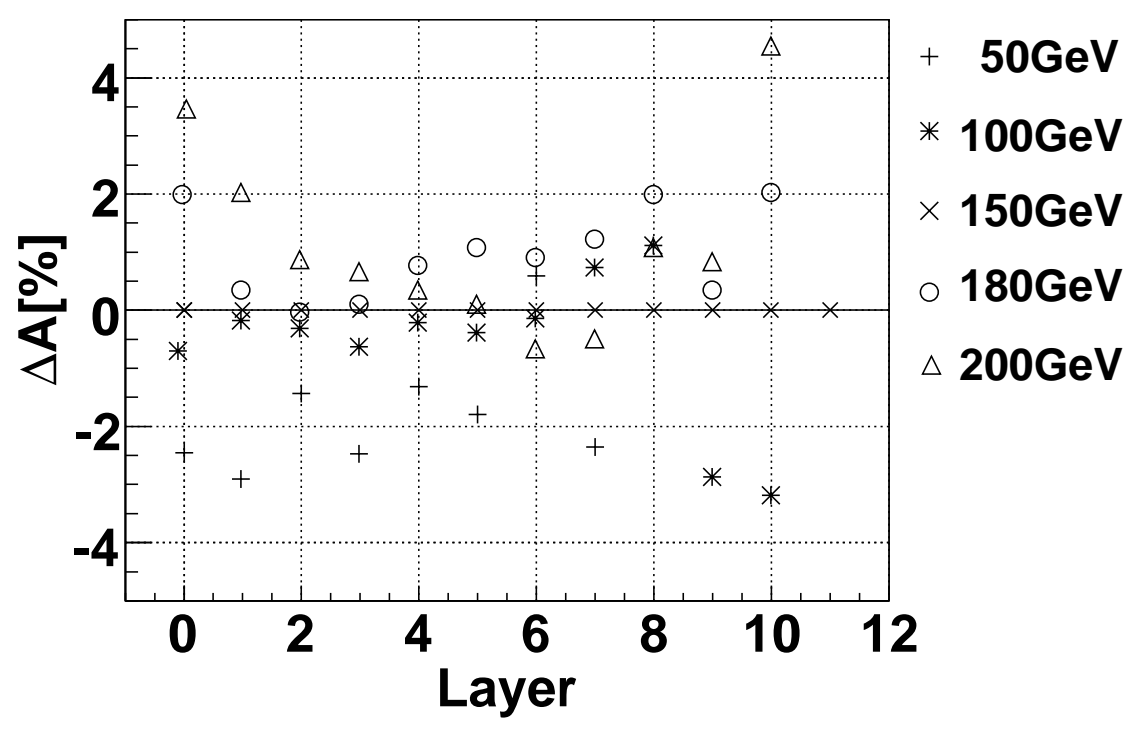

Figure 4.10: Comparison of gain conversion factors obtained from electron showers of different energy. Here the relative differences from the $150 \mathrm{GeV}$ beam are shown for each layer.

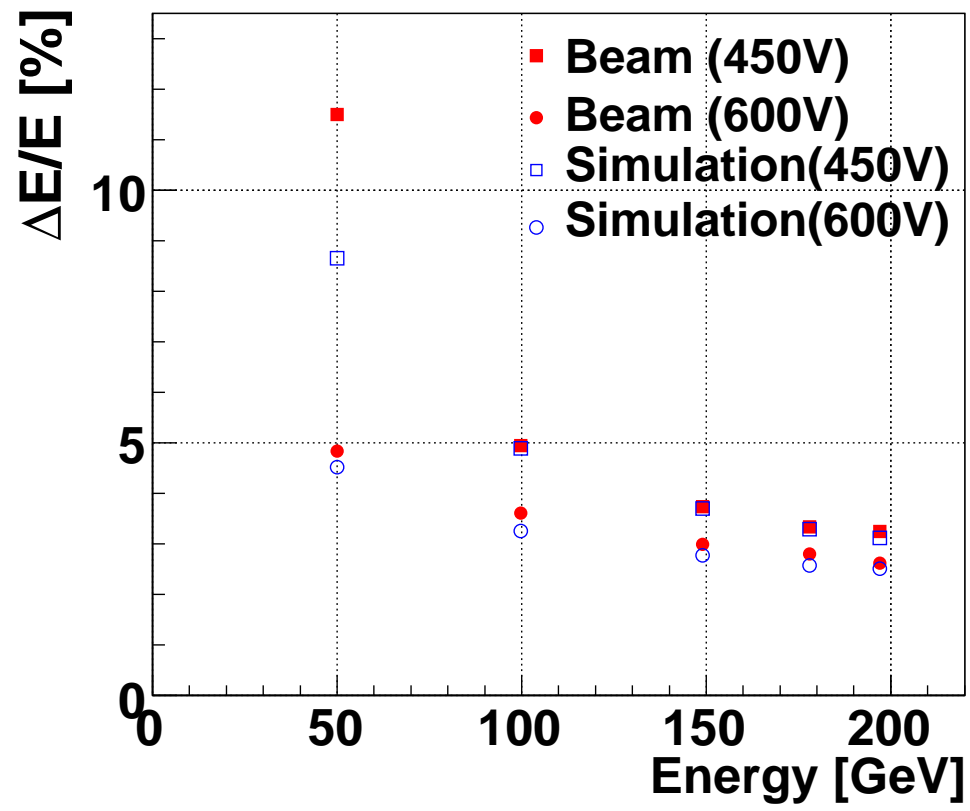

Figure 4.11: Energy resolution for electrons with two different bias HV values are given. The results of MC simulation are also plotted with blue circles.

\subsubsection{Position resolution of the SciFi}

The position resolution of the SciFi hodoscope used in detector 1 has been studied by using the lateral spread of electron showers at $6 \mathrm{X}_{0}$ inside the calorimeter. The position of each SciFi is calibrated beforehand by detecting a muon track which is also detected by ADAMO placed in front of the detector [22]. The center of the shower is determined from the peak signal and the signals in 

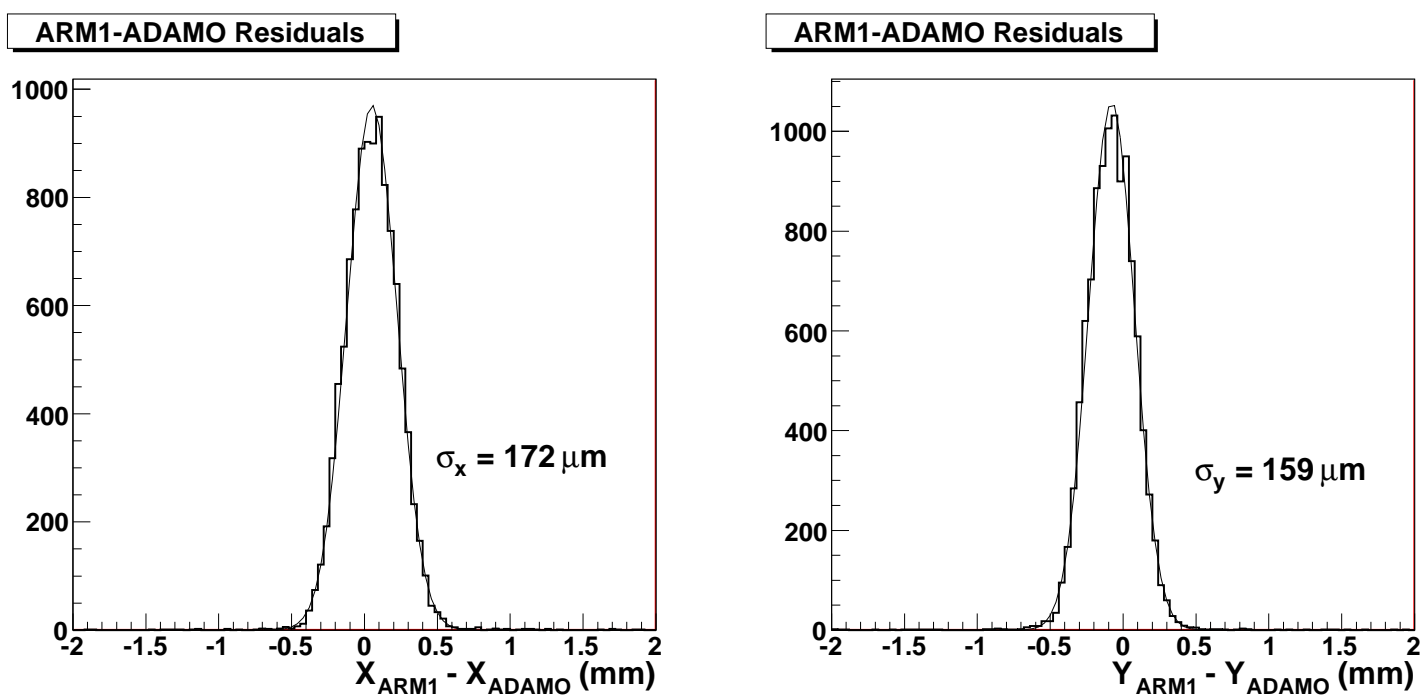

Figure 4.12: Position resolution of the SciFi in detector 1. Distributions of the difference between the shower center and incident particle position determined by SciFi and ADAMO, respectively, are plotted. The results for $\mathrm{X}$ and $\mathrm{Y}$ directions are shown in the left and right plots, respectively.

the two adjacent fibers weighted by their AD counts. The center determined by the SciFi hodoscope is compared with the incident particle position estimated by ADAMO. The distributions of the differences between these measurements in the $\mathrm{X}$ and $\mathrm{Y}$ directions are presented in figure 4.12 for $200 \mathrm{GeV}$ electrons observed by the $20 \mathrm{~mm} \times 20 \mathrm{~mm}$ tower. The electron beams were uniformly scanned over the surface of the calorimeter, and the events $>2 \mathrm{~mm}$ from the nearest edge were selected for this analysis. The distributions can be fit by Gaussian distributions with a standard deviation of $\sigma_{x}=170 \mu \mathrm{m}$ and $\sigma_{y}=160 \mu \mathrm{m}$. The same analysis has been carried out for electron beams of 50,100,150 and $180 \mathrm{GeV}$. The resolution becomes slightly better with increasing energy from $200 \mu \mathrm{m}$ to $170 \mu \mathrm{m}$ in the $\mathrm{X}$ direction and from $210 \mu \mathrm{m}$ to $160 \mu \mathrm{m}$ in the $\mathrm{Y}$ direction. The analysis for the $40 \mathrm{~mm} \times 40 \mathrm{~mm}$ tower was done at an electron beam energy of $100 \mathrm{GeV}$, and the resolution obtained was $170 \mu \mathrm{m}$ in the $\mathrm{X}$ direction and $230 \mu \mathrm{m}$ in the $\mathrm{Y}$ direction. The results are very close to the $0.2 \mathrm{~mm}$ desired position resolution for energy above $100 \mathrm{GeV}$. Mis-alignment information between the LHCf detector and ADAMO has not yet been taken into account, hence an improvement in resolution is expected.

\subsubsection{Position resolution of the silicon tracker}

The silicon based tracker of detector 2 allows for a very good reconstruction of the $\gamma$-ray impact point on the calorimeter, that improves with increasing the $\gamma$-ray energy. From a MC simulation of the system, we calculated an expected impact point reconstruction resolution better than $100 \mu \mathrm{m}$ for photon energy greater than $100 \mathrm{GeV}$.

A very preliminary data analysis has been done to give an estimate of the spatial resolution for the silicon based system of detector 2. This analysis was done using ADAMO for the reconstruction of the trajectory of each particle hitting the calorimeter. ADAMO was designed measuring the trajectories of single minimum ionizing particles. 


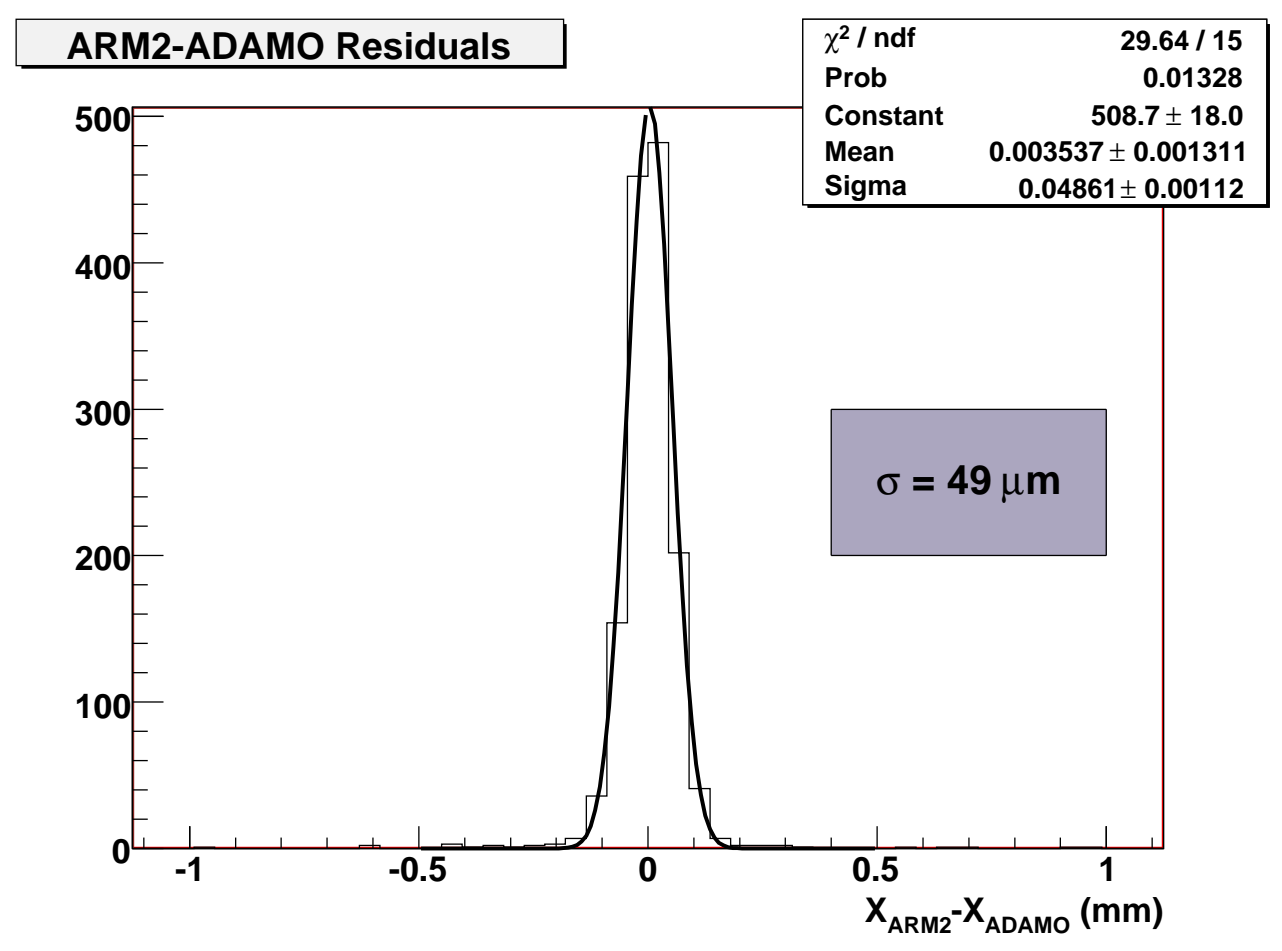

Figure 4.13: Position resolution of the silicon strip sensor in detector 2. Distribution of the differences between the measured positions of the shower centers of $200 \mathrm{GeV}$ electrons at $6 \mathrm{X}_{0}$ inside the calorimeter and the impact points extrapolated at the same depth by using the ADAMO tracker.

Figure 4.13 shows the distribution of the differences between the measured positions of the shower centers on the LHCf silicon layer located at $6 \mathrm{X}_{0}$ inside the calorimeter and the impact points extrapolated at the same depth by using the ADAMO tracker. A data set of $200 \mathrm{GeV}$ electrons events has been used for this analysis. Because the ADAMO system has an intrinsic spatial resolution of a few $\mu \mathrm{m}$ for minimum ionizing particles, the width of this distribution is dominated in good approximation by the LHCf silicon spatial resolution. These first results show therefore that the center of a $200 \mathrm{GeV}$ e.m. shower can be reconstructed on the silicon layer located at $6 \mathrm{X}_{0}$ inside the calorimeter with a spatial resolution of about $50 \mu \mathrm{m}$.

\subsubsection{Demonstration of $\pi^{0}$ mass reconstruction capability}

To demonstrate the capability of the LHCf detectors for $\pi^{0}$ mass reconstruction, an experiment was performed at the SPS H4 test beam with detector 1 . To produce $\pi^{0}$ 's, a carbon target of $60 \mathrm{~mm}$ thickness was placed $10 \mathrm{~m}$ in front of the detector and the target was exposed to a $350 \mathrm{GeV}$ proton beam. With this geometry and energy, we can expect typically $20 \mathrm{GeV} \gamma$-ray pairs entering in the calorimeters. The PMTs were operated at $800 \mathrm{~V}$. With this voltage the gain is 40 times higher than the gain at the nominal voltage of $450 \mathrm{~V}$. To trigger on gamma-ray pairs, the trigger logic was set so that both calorimeters were required to have signals corresponding to more than 40-80 MIPS in 


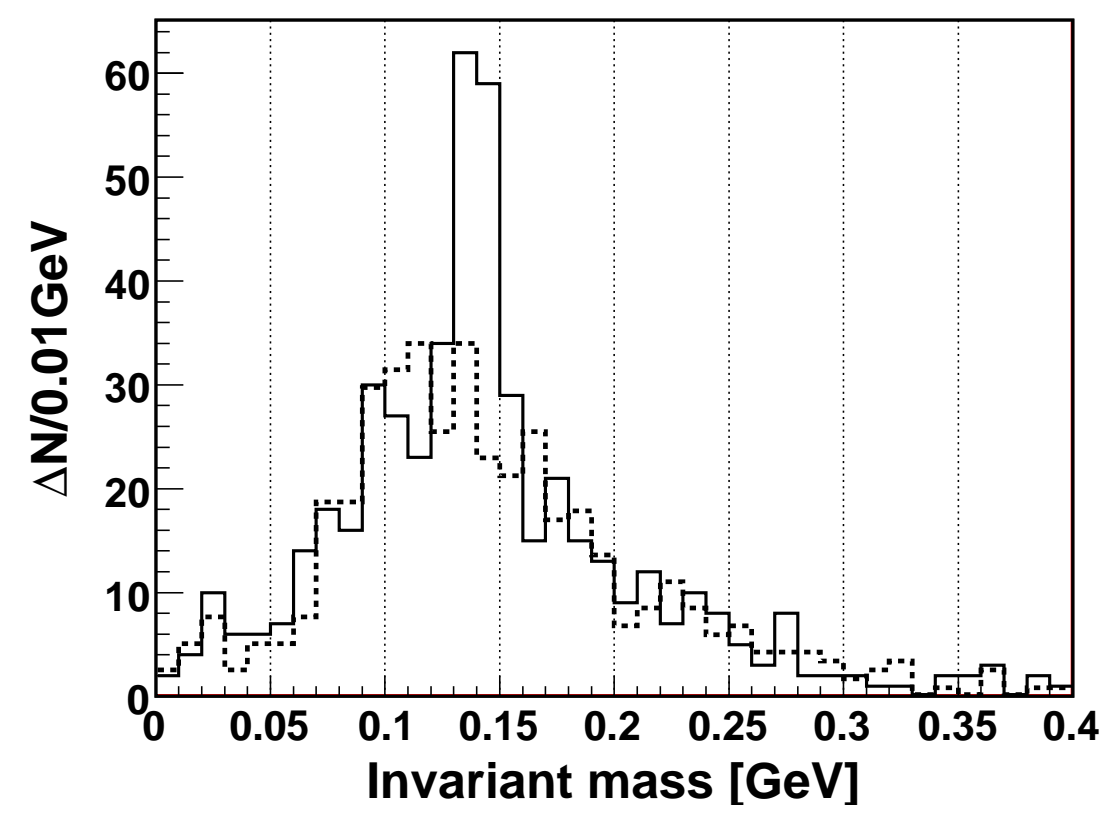

Figure 4.14: Invariant mass distribution calculated from the $\gamma$-ray pairs observed in the SPS test beam. A clear peak at the mass of $\pi^{0}(135 \mathrm{MeV})$ implies the calibration and analysis procedure were working correctly. The background shown in the dashed histogram was calculated by using uncorrelated $\gamma$-ray pairs.

any of the layers from 2 to 5 . At the same time, to reduce the large proton background, we required that none of the last two scintillation layers in both calorimeters have a signal exceeding 20 MIPs.

After $6 \times 10^{7}$ protons entered the carbon target, 300000 events generated the trigger and were recorded. Data reduction was carried out based on the processes described in the previous sections, i.e., position determination based on the SciFi data, $2 \mathrm{~mm}$ edge cut, correction for non-uniform light collection, leakage correction, and conversion from measured signals to energy deposited. The difference between the PMT gain in the nominal beam tests and in this special run was also accounted for based on the laser calibration data. Clear $\gamma$-ray pairs, each having energy more than $20 \mathrm{GeV}$, are identified in $0.6 \%$ of these recorded events. From the energies and the transverse positions of the pair, the invariant mass was calculated. The invariant mass distribution is shown in figure 4.14. A clear peak at the mass of the $\pi^{0}(135 \mathrm{MeV})$ implies the calibration and analysis procedure are working correctly. The background is caused by uncorrelated pairs those accidentally hit the two calorimeters simultaneously. The background distribution was evaluated by shifting the events in the two calorimeters so that any correlated pairs disappear. The result is also shown in figure 4.14 by dashed line and confirms the peak is really produced by $\pi^{0}$ 's. Here we note that the energy range of $\gamma$-rays in this test $(20-50 \mathrm{GeV})$ was below the range that will be investigated in LHCf $(>100 \mathrm{GeV})$. 


\subsection{Results of radiation damage tests}

The LHCf detectors will be exposed to a considerable amount of ionizing radiation. Mokhov et al. (2003) calculated the absorbed dose distribution around the TAN [23]. Their result showed that the LHCf detector would receive a maximum radiation dose of $10 \mathrm{~Gy} /$ day at a nominal luminosity of $10^{29} \mathrm{~cm}^{-2} \mathrm{~s}^{-1}$. We have tested the tolerances of our plastic scintillators and clear-fiber lightguides to ionizing radiation. The test experiments were carried out with a heavy ion beam from a synchrotron at HIMAC of NIRS (National Institute of Radiological Science, Japan) and with $\gamma$ rays at the ${ }^{60}$ Co Radiation Facility of Nagoya University. Effects of irradiation such as a change in the light output from the plastic scintillators and change in transparency of the light guides for the scintillation photons and also the recovery from radiation damage with time were measured.

For the heavy ion test, two kinds of plastic scintillator (EJ-260 and BC-404) and a clearfiber light-guide (CLEARPSM, Kuraray) were irradiated with $290 \mathrm{MeV} / \mathrm{n}{ }^{12} \mathrm{C}$ ion beams. Absorbed radiation dose was measured by an air ionization chamber coupled with measurements by plastic scintillators. The final integrated dose was $30 \mathrm{kGy}$ with a beam intensity of $10^{8}$ particles $\mathrm{s}^{-1} \mathrm{~cm}^{-2}$. The degradation of scintillator light outputs were evaluated by measuring the signal for the weak ${ }^{12} \mathrm{C}$ beam itself and for UV light $(350 \mathrm{~nm}$ ) from a filtered Xe flash lamp (Hamamatsu L4633-01). Different irradiation rates $\left(3 \times 10^{4} \mathrm{~Gy} / \mathrm{h}, 8 \times 10^{4} \mathrm{~Gy} / \mathrm{h}\right.$ and increasing rate from $2 \times 10^{0}$ to $8 \times 10^{4} \mathrm{~Gy} / \mathrm{h}$ ) were compared. For $\gamma$-ray tests, similar materials were irradiated by a $100 \mathrm{TBq}$ ${ }^{60} \mathrm{Co}$ source. The absorbed dose was calculated from the geometry of the source and target materials. Two dose rates $(22 \mathrm{~Gy} / \mathrm{h}$ and $250 \mathrm{~Gy} / \mathrm{h})$ were used by changing the distance between the source and the irradiated materials. The evaluation of the light output was done by using UV light from the Xe flash lamp. The short-term recovery of damage was also measured just after irradiation by both heavy ions and $\gamma$-rays. The long-term recovery was measured by using a radioactive beta-ray source $\left({ }^{90} \mathrm{Sr}\right)$ for several days or more. Figure 4.15 shows the variation of the scintillator and light guide light outputs for irradiation by heavy ions and $\gamma$-rays. The scintillator light outputs are almost constant up to ten Gy of absorbed dose and then decrease to $90 \%$ of the pre-irradiation value at 100 Gy or more. Most samples show a decrease of light output to $40 \%$ of the pre-irradiation value at the maximum absorbed dose of $30 \mathrm{kGy}$. There was no significant difference in the effects of irradiation measured by UV light or by the ${ }^{12} \mathrm{C}$ beam. Irradiation of the fiber light-guide showed a small decrease of light transmission for the wavelength of $490 \mathrm{~nm}$ because of the decrease in transparency due to irradiation. The measurement for irradiation by $\gamma$-rays showed a decrease of light output similar to that for heavy ions. The light output decreases to $90 \%$ of the initial value around $100 \mathrm{~Gy}$ and to $80 \%$ at $500 \mathrm{~Gy}$. The damaged scintillators showed a tendency to recover within a few hours after finishing the irradiation, but the extent of recovery was very small. After one day or more, a clear recovery was observed and the light output increased from $\sim 40 \%$ to $~ 60 \%$ of the initial light output before irradiation.

In conclusion, the plastic scintillators can be damaged by irradiation with heavy ions and $\gamma$ rays but the decrease of the light output is not large for doses of interest for LHCf. No significant dependence of radiation damage on the type of scintillator, the type of radiation, the irradiation rate, and so on were observed. Partial recovery of decreased light output is observed over several days after irradiation. Normally, the LHCf detectors will receive a dose of several tens of Gy during one week oepration. This is enough to complete the measurements unless background event rates 


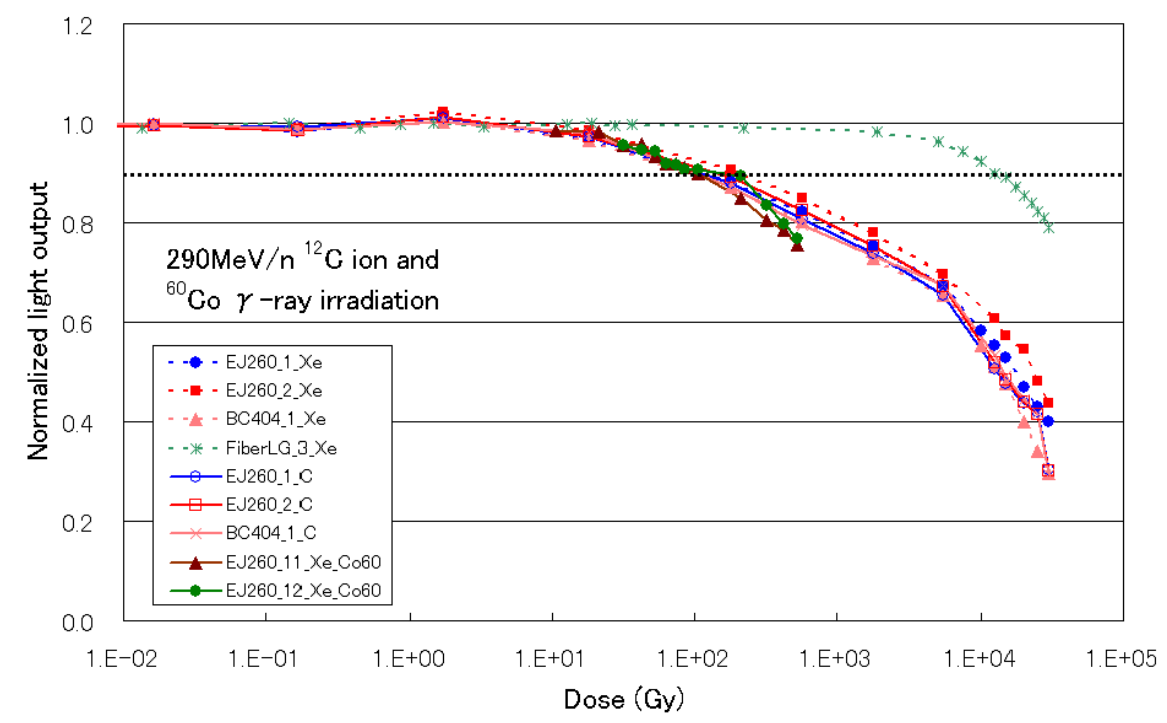

Figure 4.15: The variation of the scintillator light outputs (EJ260 and BC404) and light guide transmission for irradiation by heavy ions and $\gamma$-rays.

are very high and the run must be extended. A small decrease of light output can be corrected for by the laser calibration system and the accuracy of energy measurement will not be affected. 


\section{Chapter 5}

\section{Summary}

The LHCf is an experiment to measure very forward neutral particles emitted in LHC collisions in order to calibrate the hadron interaction models used in simulation of Extremely High Energy Cosmic-Rays at a laboratory equivalent energy of $10^{17} \mathrm{eV}$. In this paper, we have given an experimental overview, a description of details of the instrumentation and data acquisition system, simulation of performance of the detectors during LHC operation and reported preliminary results of SPS test beam experiments.

Two LHCf detectors are installed at $\pm 140 \mathrm{~m}$ from IP1 where the neutral particle absorbers (TAN) are located. Each of the two detectors is composed of two independent sampling calorimeters made of tungsten plates, plastic scintillators and position sensitive layers. The position sensitive layers are scintillation fibers and multi-anode photomultipliers for detector 1 and silicon strip detectors for detector 2. Because of the limited space for installation, these calorimeters are very compact, but are still capable of measuring energy and position of the incident particles with sufficient resolutions. Simulations predict that the energy resolution is better than $5 \%$ and the position resolution as good as $0.2 \mathrm{~mm}$ at $100 \mathrm{GeV}$; these predictions have been confirmed by measurements in SPS test beams at CERN. The use of front scintillation counters is expected to help reduce background events generated by beam-gas interactions during early LHC operation when vacuum conditions may not be as good as anticipated. The detectors are equipped with vertical manipulators to increase the $\mathrm{P}_{T}$ range that can be measured and to remove the detectors from unnecessary radiation damage when they are not in use. The experiment covers a pseudo rapidity range from 8.4 to infinity. Data acquisition during LHC operation will be carried out in the ATLAS counting room, USA15. An event tagging scheme based on the time stamp of the LHC clock has been developed to correlate LHCf and ATLAS events for possible further analysis. Detectors, data acquisition and electronics are optimized to operate during the early phases of LHC commissioning with luminosity below $10^{30} \mathrm{~cm}^{-2} \mathrm{~s}^{-1}$. The event rates are expected to be high enough that LHCf can get all of its data in one week of LHC operation at the luminosity of $10^{29} \mathrm{~cm}^{-2} \mathrm{~s}^{-1}$. After a week of operation under these conditions, the output of the plastic scintillators will be degraded by $\sim 10 \%$ due to radiation damage. Radiation damage will be monitored and corrected for with a pulsed laser calibration system. The LHCf detectors will be removed once the luminosity exceeds $10^{30} \mathrm{~cm}^{-2} \mathrm{~s}^{-1}$. 


\section{Acknowledgments}

This work is partly supported by Grant-in-Aid for Scientific Research (B:16403003), Grant-in-Aid for Scientific Research on Priority Areas (Highest Cosmic Rays: 15077205, 1733004, 19012003) and Grant-in-Aid for Young Scientists (B:18740141), by the Ministry of Education, Culture, Sports, Science and Technology (MEXT) of Japan. This work is also partially supported by the Mitsubishi Foundation in Japan and by Istituto Nazionale di Fisica Nucleare (INFN) in Italy. The receipt of a Japan Society for the Promotion of Science (JSPS) Research Fellowship (H.M.) is also acknowledged. The authors would like to thank CERN for the support shown to our experiment. 


\section{Bibliography}

[1] M. Takeda et al., Extension of the cosmic ray energy spectrum beyond the predicted Greisen-Zatsepin-Kuz'min cutoff, Phys. Rev. Lett. 81 (1998) 1163.

[2] S. Yoshida et al., Extremely high-energy neutrinos and their detection. Astrophys. J. 479 (1997) 547.

[3] R.U. Abbasi et al., Measurement of the Flux of Ultrahigh Energy Cosmic Rays from Monocular Observations by the High Resolution Fly's Eye Experiment, Phys. Rev. Lett. 92 (2004) 151101.

[4] T. Yamamoto et al., The UHECR spectrum measured at the Pierre Auger Observatory and its astrophysical implications, Proc. of $30^{\text {th }}$ Intl. Cosmic Ray Conference, Merida Mexico (2007).

[5] The Pierre Auger Collaboration, Correlation of the Highest-Energy Cosmic Rays with Nearby Extragalactic Objects, Science 318 (2007) 938.

[6] M. Unger et al., Study of the Cosmic Ray Composition above 0.4 EeV using the Longitudinal Profiles of Showers observed at the Pierre Auger Observatory, Proc. of $30^{\text {th }}$ Intl. Cosmic Ray Conference, Merida Mexico (2007).

[7] The TOTEM collaboration, The TOTEM Experiment at the CERN Large Hadron Collider, 2008 JINST 3 S08007.

[8] The ATLAS collaboration, ATLAS Forward Detectors for Measurement of Elastic Scattering and Luminosity, CERN-LHCC-2008-004, http://cdsweb.cern.ch/record/1095847.

[9] E. Paré et al., Inclusive production of $\pi^{0}$ 's in the fragmentation region at the SppS collider, Phys. Lett. B 242 (1990) 531.

[10] T. Sako et al., Performance of the prototype detector for the LHCf experiment, Nucl. Instrum. Meth. A 578 (2007) 146.

[11] The ATLAS collaboration, Zero Degree Calorimeters for ATLAS, CERN-LHCC-2007-001, http://cdsweb.cern.ch/record/1009649.

[12] P. Datte et al., Initial test results of an ionization chamber shower detector for a LHC luminosity monitor, IEEE Trans. Nucl. Sci. 50 (2003) 258. 
[13] http://atlas.web.cern.ch/Atlas/GROUPS/INNER_DETECTOR/SCT/, following the link for the barrel module.

[14] P. Aspell et al., PACE3: a large dynamic range analog memory front-end ASIC assembly for the charge readout of silicon sensors, IEEE Nucl. Sci. Symp. Conf. Rec. 2 (2005) 904.

[15] L. Bonechi et al., Production and test of the LHCf microstrip silicon system, Presented at the RD07 Conference, Florence Italy, June 27-29 2007.

[16] MIDAS Homepage, https://midas.psi.ch/

[17] ROOT Homepage, http://root.cern.ch/

[18] J. Varela eds., CMS L1 Trigger Control System, CMS-NOTE-2002-033, http://cdsweb.cern.ch/record/687458.

[19] CMS collaboration, V. Karimäki, CMS Tracker Technical Design Report, CERN-LHCC-98-006, CMS-TDR-005, http://cdsweb.cern.ch/record/368412.

[20] K. Kasahara, EPICS Homepage, http://cosmos.n.kanagawa-u.ac.jp/.

[21] J. Ranft, Dual parton model at cosmic ray energies, Phys. Rev. D 51 (1995) 64.

[22] L. Bonechi et al., Development of the ADAMO detector: test with cosmic rays at different zenith angles, Proc. of $29^{\text {th }}$ Intl. Cosmic Ray Conference, Pune India, 9 (2005) 283, http://icrc2005.tifr.res.in/htm/Vol-Web/Volume9_index.html.

[23] N.V. Mokhov et al., Protecting LHC IP1/IP5 Components Against Radiation Resulting from Colliding Beam Interactions, LHC-Project-Report-633 (2003), http://cdsweb.cern.ch/record/613167. 